\title{
28 Research Suare \\ Deep learning-based brain age prediction in normal aging and dementia
}

Jeyeon Lee

Mayo Clinic

Brian Burkett

Mayo Clinic

Hoon-Ki Min

Mayo Clinic

Matthew Senjem

Mayo Clinic https://orcid.org/0000-0001-9308-9275

Emily Lundt

Mayo Clinic

Hugo Botha

Mayo Clinic

Jonathan Graff-Radford

Mayo Clinic

Leland Barnard

Mayo Clinic

Jeffrey Gunter

Mayo Clinic

Christopher Schwarz

Mayo Clinic https://orcid.org/0000-0002-1466-8357

Kejal Kantarci

Mayo Clinic

David Knopman

Mayo Clinic

Bradley Boeve

Mayo Clinic

Val Lowe

Mayo Clinic

Ronald Petersen

Mayo Clinic

Clifford Jack

Mayo Clinic Hospital https://orcid.org/0000-0001-7916-622X

David Jones ( $\sim$ Jones.david@mayo.edu ) 
Mayo Clinic

\section{Article}

Keywords: Deep learning, Convolutional neural network, Brain age, Brain age gap, Structural MRI, FDG PET, Saliency map, Dementia, Alzheimer's disease

Posted Date: August 24th, 2021

DOl: https://doi.org/10.21203/rs.3.rs-804454/v1

License: (c) (i) This work is licensed under a Creative Commons Attribution 4.0 International License. Read Full License

Version of Record: A version of this preprint was published at Nature Aging on May 9th, 2022. See the published version at https://doi.org/10.1038/s43587-022-00219-7. 
1 Deep learning-based brain age prediction in normal aging and dementia

3 Jeyeon Lee ${ }^{1}$, Brian J. Burkett ${ }^{1}$, Hoon-Ki Min ${ }^{1}$, Matthew L. Senjem², Emily S. Lundt ${ }^{3}$, Hugo

4 Botha $^{4}$, Jonathan Graff-Radford ${ }^{4}$, Leland R. Barnard ${ }^{4}$, Jeffrey L. Gunter ${ }^{1}$, Christopher G.

5 Schwarz ${ }^{1}$, Kejal Kantarci ${ }^{1}$, David S. Knopman ${ }^{4}$, Bradley F. Boeve ${ }^{4}$, Val J. Lowe ${ }^{1}$, Ronald

6 C. Petersen ${ }^{4}$, Clifford R. Jack Jr. ${ }^{1}$, David T. Jones ${ }^{4 *}$

$8 \quad{ }^{1}$ Department of Radiology, Mayo Clinic, Rochester, MN, USA

$9 \quad{ }^{2}$ Department of Information Technology, Mayo Clinic, Rochester, MN, USA

$10{ }^{3}$ Department of Health Sciences Research, Mayo Clinic, Rochester, MN, USA

$11{ }^{4}$ Department of Neurology, Mayo Clinic, Rochester, MN, USA

$12{ }^{*}$ Correspondence to: David T. Jones M.D., Mayo Clinic, Department of Neurology, 200

13 First Street SW, Rochester, Minnesota, 55905, USA

14 E-mail: Jones.david@mayo.edu

16 Keywords: Deep learning; Convolutional neural network; Brain age; Brain age gap;

17 Structural MRI; FDG PET; Saliency map; Dementia; Alzheimer's disease.

\section{Abstract}

20 Normal brain aging is accompanied by patterns of functional and structural change.

21 Alzheimer's disease (AD), a representative neurodegenerative disease, has been linked

22 to accelerated brain aging at respective age ranges. Here, we developed a deep

23 learning-based brain age prediction model using fluorodeoxyglucose (FDG) PET and 
24 structural MRI and tested how the brain age gap relates to degenerative cognitive

25 syndromes including mild cognitive impairment, $A D$, frontotemporal dementia, and Lewy

26 body dementia. Occlusion analysis, performed to facilitate interpretation of the model,

27 revealed that the model learns an age- and modality-specific pattern of brain aging. The

28 elevated brain age gap in dementia cohorts was highly correlated with the cognitive

29 impairment and AD biomarker. However, regions generating brain age gaps were

30 different for each diagnosis group of which the $A D$ continuum showed similar patterns to

31 normal aging in the $\mathrm{CU}$.

\section{Introduction}

34 The biology of aging is complex ${ }^{1}$ and has yet to be fully understood. ${ }^{2}$ In general, aging

35 is characterized by the gradual accumulation of deleterious biological changes

36 accompanying a progressive loss of function ${ }^{1}$, although this is not an all-encompassing

37 definition. The endeavor to better understand the biology of the aging brain is widely

38 relevant as the impact of aging on the human brain and associated changes in cognitive

39 function have implications for quality of life in the elderly.

41 The aging of the brain entails both structural and functional changes. Structural

42 magnetic resonance imaging (MRI) has shown that increased age is associated with

43 reduction of grey matter volume, most prominently in the frontal lobes, insular cortex,

44 and hippocampus ${ }^{3-6}$, increased volume of the ventricular system and intracranial

45 cerebrospinal fluid ${ }^{3,4,7}$, and changes in white matter microstructure. ${ }^{7,8}$ In addition,

46 functional imaging techniques using positron emission tomography (PET) have shown 
47 that brain aging is associated with decreased global oxygen utilization, cerebral blood

48 flow, glucose uptake, and regional changes in aerobic glycolysis. ${ }^{9,10}$ Age-related

49 decreased glucose utilization has been found most prominently in the frontal lobes,

50 posterior cingulate, and posterior parietal lobes. ${ }^{11-13}$ The temporal lobe, including medial

51 temporal regions - a critical area of pathology in dementia - has also showed an age-

52 dependent decrease in glucose metabolism. ${ }^{14-16}$ In contrast, the primary motor cortex,

53 occipital cortex, cerebellum and sub-cortical structures including thalamus, putamen,

54 and pallidum have been found to be less susceptible to metabolic changes with aging. ${ }^{17}$

56 Based on these findings, age prediction using brain imaging is an active area of

57 neuroscience research. ${ }^{18-22}$ An estimated age can be referred to as "brain age" for an

58 individual which may differ from the individual's chronological age. ${ }^{19}$ Recently, growth in

59 data availability and advancement of deep learning (DL) techniques have allowed more

60 accurate brain-age estimation in the cognitively normal population through convolutional

61 neural network (CNN) models. ${ }^{21-25}$ In addition, the 'brain age gap', which is the

62 difference between the 'predicted brain age' and 'chronological age', has been found to

63 be useful as a promising, personalized biomarker of brain health. ${ }^{19}$ On an individual

64 basis, brain age gap measurements may also prove to have prognostic value,

65 potentially predicting health outcomes by capturing individual differences in the

66 interaction of aging and disease..$^{19}$ Several studies have reported that an over-

67 estimation of an individual's chronologic age based on a prediction from neuroimaging,

68 measured as a large brain age gap, is associated with mortality ${ }^{26}$, neurodegenerative

69 diseases $^{27}$ and various other clinical conditions. ${ }^{19,20}$ Moreover, measuring the brain age 
70 gap in cases of neurodegenerative pathology may inform our understanding of disease

71 risk, resilience to structural/functional insults which accumulate with aging, and the

72 effects of diseases on the aging brain. For example, Alzheimer's disease has been

73 linked to accelerated brain aging at respective age ranges ${ }^{28,29}$, implying that dementia is

74 an extreme phenotype of the aging process. Thus, a reliable measure of typical brain

75 aging may be beneficial in order to better distinguish from pathological aging. ${ }^{30}$

77 We aimed to develop a deep-learning-based brain age prediction model using a large

78 collection of brain structural MRI and Fluorodeoxyglucose (FDG) PET scans from

79 participants $20-98$ years old ( $n=2,349$ unique individuals with 4,127 brain scans;

80 cognitively unimpaired (CU) normal controls $=1,805$ and cognitively impaired $=732$ ).

81 Our brain age prediction method was developed using the images from only the CU

82 participants to train the healthy aging trajectories. We also studied age- and modality-

83 specific saliency maps of the CNN model explaining which brain regions contribute most

84 to age prediction for each age subgroup and modality type using an occlusion sensitivity

85 analysis. We then investigated the brain age gap estimation in the patient groups

86 including mild cognitive impairment (MCl), Alzheimer's disease (AD), Frontotemporal

87 Dementia (FTD), and Dementia with Lewy Bodies (DLB). We evaluated for associations

88 of brain age gap with neuropsychological tests and other imaging AD biomarkers, such

89 as amyloid PET and tau PET. We then performed a voxel-wise linear regression

90 analysis to look at which regional alterations contribute to higher brain age gap

91 generation for each disease group and compared them with normal brain aging

92 trajectories. 


\section{Results}

95 Brain age estimation in CU participants. Our brain age prediction model based on

96 FDG PET or MRI was trained on CU participants in the Mayo dataset $(n=1,805)$ using

97 a 3D Densenet architecture (Fig. 1A). ${ }^{31}$ For the training, we only utilized scans of the

98 first time point per a participant to avoid possible data leakage between the training and

99 validation/test sets. Then, the models were applied for predicting the brain age and the

100 accuracies were evaluated as a mean absolute error (MAE) with 5-fold cross validation.

101 Fig. 2 illustrates the scatterplots of the test set predictions against chronological age.

102 The result showed that our FDG- and MRI-based model could accurately predict the 103 chronological age of healthy adults $\left(R^{2}=0.8546\right.$ and beta $=0.8503$ for FDG and $R^{2}=$

1040.8046 and beta $=0.7718$ for $\mathrm{MRI})$. The overall performance measured on the test set

105 was MAE $=3.4333 \pm 0.0545$ and $4.2055 \pm 0.2241$ for FDG and MRI, respectively

106 (Supplementary table 2). As shown in Fig. 2b and e illustrating the scatterplot of brain

107 age gap (predicted brain age-chronological age) as a function of corresponding

108 chronological age, the estimation results showed a tendency to be biased towards the

109 mean age of the total cohort, resulting in a negative correlation between the brain age

110 gap and chronological age (Spearman's $r=-0.3613$ and -0.4642 for FDG and MRI,

111 respectively). This phenomenon is well-known to be associated with regression dilution

$112{ }^{32}$, model regularization and a non-Gaussian age distribution. ${ }^{33}$ We used a linear bias

113 correction method ${ }^{33}$ for age bias correction for the brain age gap. After the bias

114 correction, we observed that the correlation between the corrected brain age gap and

115 chronological age decreased to 0.0396 and 0.0303 for FDG and MRI respectively, and 
116 MAE also decreased to 3.1212 and 3.3669 for FDG and MRI (Fig. 2c and f). The overall

117 performance after bias correction for total fold was MAE $=3.0755 \pm 0.1401$ and 3.4868

$118 \pm 0.1631$ for FDG and MRI, respectively (Supplementary table 2).

119 To assess whether the trained model presents a dataset-specific bias, the model trained 120 with Mayo dataset was applied to an independent cohort, the Alzheimer's Disease

121 Neuroimaging initiative (ADNI; adni.loni.usc.edu) dataset (CU, $n=454)$. We obtained a 122 comparable result (corrected test MAE $=2.8942$ for $F D G$ and corrected test MAE $=$ 1233.5766 for MRI), implying that the models were fairly generalizable to the independent 124 dataset (Supplementary Fig. 1 and supplementary table 2). In addition, we also trained 125 a model by blending Mayo and ADNI dataset together (Supplementary Fig. 2). In this 126 trial, the overall performance of age prediction was better than using Mayo dataset only 127 (corrected test MAE $=2.7383 \pm 0.1091$ for FDG and corrected test MAE $=3.1029 \pm$ 1280.2107 for MRI; supplementary table 2).

129 To examine how the data-split option considering inter-participant variability and within130 participant variability affects performance, the prediction accuracies of several data-split 131 strategies were compared (as detailed in the methods section; Supplementary table 3).

132 Expectedly, we observed that the overlap of participants between the training dataset 133 and validation or test set significantly affected the accuracy of age estimation (option 2 134 and option 3 in supplementary table 3). This pattern was similarly found in both FDG 135 and MRI. On the other hand, whether to include multiple time points for each participant 136 has minimal effects on the model's performance (option 4 and option 5 in 137 supplementary table 3$)$. 
139 Age- and modality-specific saliency map of brain age prediction model. For an

140 interpretability of trained 3D-Densenet model, the saliency maps for age subgroups

141 were estimated through occlusion sensitivity analysis. In the occlusion sensitivity

142 analysis method, a portion of brain in the input space was occluded with a mask

$143(11 \times 11 \times 11)$ by setting these voxels to zero, and their relevance in the decisions was

144 indirectly estimated by calculating the change of MAE (MAE $E_{\text {occlusion }}-M_{A} E_{\text {original; }}$ Fig. 1B).

145 The results revealed age- and modality-specific saliency patterns (Fig. 3 and

146 supplementary Fig. 3). For the FDG model (left panel in Fig. 3), a posterior to anterior

147 transition was observed with increased age. The overall posterior region with a peak at

148 the posterior cingulate cortex had a higher contribution for age prediction in the younger

149 group (30-40 and 40-50 years). Meanwhile, for the 50-60 and 70-80 years of age

150 groups, the inferior frontal regions including the orbitofrontal and olfactory cortex were

151 dominantly utilized for age prediction. Prefrontal regions also showed a higher

152 contribution than other areas. A global contribution with the peak around the inferior

153 frontal cortex and basal ganglia was also found to be important for age prediction in the

154 older group (80-90 and 90-100 years). For MRI (right panel in Fig. 3), the insular cortex

155 contributed most to age prediction in the younger group (30-40 and $40-50$ years). From

156 50-60 years, the ventricular boundary showed a higher contribution. The

157 cerebellomedullary cistern showed the highest saliency in the older groups (80-90 and $158 \quad 90-100$ years).

160 Brain age gap estimation in patient groups. The brain age gap of four clinical

161 diagnosis groups (MCl, AD, FTD, and DLB) was estimated using the 3D-Densenet 
162 model trained with normative cohorts. Fig. 4 illustrates the scatterplot of brain age gap

163 against chronological age for each patient group. The brain age gap was corrected

164 using the same coefficients used for bias correction of CU (Fig. 2). As expected, the

165 brain age gap of all patient groups was significantly higher than that of $\mathrm{CU}$ group for

166 both modalities ( $P<0.001$ two-sample t-test, Fig. $4 \mathrm{e}$, and j). Interestingly, the predicted

167 brain age gap of all disease groups had a negative correlation with chronological age,

168 meaning younger patients had a higher gap. Accordingly, the mean brain age gap of

169 FTD, in which most patient was early onset, was relatively higher than that of other

170 groups, followed by $\mathrm{AD}, \mathrm{DLB}$, and $\mathrm{MCl}$ (Fig. 4e and j).

171 As shown in supplementary Fig. 4, FDG-based and MRI-based brain age gap showed

172 significant correlation with each other $(P<0.001$, Pearson's correlation, Supplementary

173 Fig. 4) in every diagnostic group. Interestingly, the disease group tended to have a

174 higher correlation and slope than the CU cohort (Pearson's correlation: 0.5819, 0.7163,

$1750.7974,0.8491$ and 0.6925 for CU, MCI, AD, FTD and DLB, respectively, slope of fitted

176 line: $0.6624,0.7080,0.8102,0.8132$ and 0.8126 for $\mathrm{CU}, \mathrm{MCl}, \mathrm{AD}, \mathrm{FTD}$ and $\mathrm{DLB}$,

177 respectively).

179 An association of brain age gap in dementia with normal aging. Then, a voxel-wise

180 linear regression analysis was performed using the brain age gap as a regressor to

181 investigate which brain regions' alteration were related to higher brain age gap

182 generation for each patient group. In this analysis, chronological age was specified as a

183 nuisance covariate because it was negatively correlated with the brain age gap. As

184 illustrated in Fig. 5, FDG and MRI-based brain age gap showed different patterns 
185 according to their disease group and imaging modality (using linear regression, FDR 186 corrected, $q<0.01$, Fig. 5). In FDG, $\mathrm{MCl}$ and $\mathrm{AD}$ groups showed a negative correlation

187 throughout the brain, meaning global hypometabolism was associated with a higher 188 brain age gap (left panel in Fig. 5). In the AD group, the frontal, temporal, and parietal 189 regions showed a stronger negative correlation. In contrast, significant hypometabolism

190 related to the brain age gap was observed in the frontal and temporal regions in the 191 FTD patient group. Interestingly, the occipital cortex showed a positive correlation with 192 brain age gap in the FTD group. The DLB group showed a significant negative 193 correlation in posterior and temporal regions.

194 However, MRI showed a distinctly different pattern of salient regions from FDG (right 195 panel in Fig. 5). In $\mathrm{MCl}$ and $\mathrm{AD}$, sulci and white matter showed a positive correlation, 196 and regions around the gyri and ventricles showed a negative correlation with brain age 197 gap. In contrast, a local negative correlation around the ventricles was marginally 198 observed for the FTD and DLB patient groups. To compare the observed brain age gap199 related changes with normal aging, a linear regression analysis was also performed for 200 the CU group using chronological age as a regressor (bottom row in Fig. 5). Similar to 201 the results for $\mathrm{MCl}$ and $\mathrm{AD}$, a global negative correlation was observed on FDG PET. A 202 positive correlation in sulci and white matter and a negative correlation in areas around 203 the gyri and ventricles was observed on MRI. The voxel-wise correlation analysis 204 showed that the beta map of $\mathrm{MCl}$ and $\mathrm{AD}$ were more strongly correlated with that of 205 normal aging than FTD and DLB for FDG (Pearson's correlation; 0.9389, 0.8384, 2060.6772 and 0.7239 for MCl, AD, FTD and DLB, respectively) and MRI (Pearson's 
207 correlation; $0.8002,0.7338,0.4922$ and 0.5356 for $\mathrm{MCl}, \mathrm{AD}, \mathrm{FTD}$ and DLB,

208 respectively).

210 An association of brain age gap with neuropsychological test scores and AD

211 biomarkers. As mentioned above, the high brain age gap has been found to be linked

212 to high cognitive impairments. ${ }^{19,20,25}$ In light of this, the association was tested on the

213 corrected brain age gap of disease groups with the three cognitive test scores, including

214 Clinical Dementia Rating sum of boxes (CDR-SB) ${ }^{34}$, Short Test of Mental Status

215 (STMS) $)^{35}$, and Mini-Mental State Examinations (MMSE) $)^{36}$. As expected, both FDG-

216 based and MRI-based brain age gap showed significant correlations with the three

217 scores $(P<0.001$, Pearson's correlation, Fig. 6).

218 Then, we sought to examine an association of brain age gap with neuroimaging

219 biomarkers for $A D$. AD is characterized by a pathology aggregation of beta-amyloid $(A \beta)$

220 and neurofibrillary tangles which can be captured by Pittsburgh Compound B (PiB) PET

221 and tau PET respectively. PiB and tau PET quantification was performed on meta-ROI

222 that has previously been shown to have a broad dynamic range across the normal to

223 pathological aging to AD dementia. A meta-ROI PiB PET standardized uptake value

224 ratio (SUVr) was derived from the average of the median SUVr in the prefrontal,

225 orbitofrontal, parietal, temporal, anterior cingulate, and posterior cingulate/precuneus

226 regions. ${ }^{37}$ A meta-ROI tau PET SUVr was formed from the average of the median

227 uptake in the amygdala, entorhinal cortex, fusiform, parahippocampal and inferior

228 temporal and middle temporal gyri. ${ }^{37}$ For meta-ROI PiB PET SUVr, only the $\mathrm{MCl}$ group

229 reached statistical significance. In FDG and MRI, the correlation coefficient was 
230 marginal and there was no obvious pattern of association in distribution (Fig. 7a and e).

231 Other disease groups did not show significance (Fig. 7b-d and f-h). However, meta-ROI

232 tau PET SUVr showed a significant correlation with brain age gap in the $\mathrm{MCl}$ and $\mathrm{AD}$

233 groups (Fig. 7i-j and m-n). In particular, the AD group showed a higher correlation

$234(r=0.5110$ for FDG and $r=0.6648$ for MRI, Fig. $7 j$ and $n)$. FTD and DLB patients showed

235 no significant correlation with meta-ROI tau PET SUVr.

\section{Discussion}

238 We developed a 3D DenseNet model, trained on structural and metabolic brain images,

239 that generates an accurate estimate of an individual's brain age during normal cognitive

240 aging. An occlusion analysis revealed anatomic regions critical to the model

241 performance and demonstrated an age-dependent saliency pattern of brain regions.

242 The patterns were distinct for each input imaging modality, structural MRI vs. FDG PET, 243 which is interesting given that the predictive accuracy of the FDG and MRI models were

244 similar. In cohorts with a neurological disorder, the brain age gap was larger than

245 cognitively CU individuals and significantly correlated with the cognitive score. Anatomic

246 regions with the greatest weight in generating the brain age gap, identified from the

247 voxel-wise linear regression analysis, were different for each diagnostic group. The

248 results for the $\mathrm{AD}$ continuum, $\mathrm{MCl}$ and $\mathrm{AD}$, showed close correlation to normal aging

249 compared to FTD or DLB, with an accelerated time frame in the $\mathrm{MCl}$ and $\mathrm{AD}$ groups

250 reflected by the larger brain age gap compared to normal aging.

251 Most previous brain age studies were based on structural MRI. ${ }^{21-26,38}$ To our knowledge, 252 only one prior study utilized FDG $\mathrm{PET}^{18}$, but that study was based on a non-DL method 
253 and utilized a substantially smaller cohort size $(n=205)$. The structural and functional

254 changes contributing to precise age prediction in the $\mathrm{DL}$ approach remain to be fully

255 elucidated. One of the major limitations of studies using a CNN is the interpretability of

256 the model. A limited number of structural MRI-based studies reported explanation maps

257 of the CNN model. ${ }^{21,22,24}$ Although CNN-based age prediction has provided high

258 accuracy, it is difficult to know which features are important for age estimation.

259 Furthermore, there is a dearth of knowledge regarding which brain alterations and

260 specific regional changes are associated with higher brain age gaps in patients.

262 Brain-specific prediction of age is of interest both as a component of overall biologic age

263 assessment, but also as a biomarker for age-associated neurologic diseases and

264 changes in neurologic function. In a broad sense, age prediction may help to elucidate

265 the relationship of the aging process to degenerative pathology. Is dementia a

266 consequence of a unique pathologic mechanism or instead an accelerated version of

267 normal aging? ${ }^{39}$ If dementia reflects a continuum of the underlying changes in brain

268 structure and metabolism to which all individuals are inevitably susceptible at various

269 rates, brain age-prediction based on neuroimaging may yield a better understanding of

270 different metabolic brain aging phenotypes. Alternatively, if types of dementia represent

271 entities with distinctly different mechanisms than normal aging, markers of brain age

272 may still prove useful in identifying individuals at greater risk for developing these

273 conditions. ${ }^{39,40}$

274 
275 Our model was able to precisely estimate an individual's chronological age based on

276 structural and metabolic neuroimaging data (corrected MAE $=3.0755 \pm 0.1401$ and

$2773.4868 \pm 0.1631$ for FDG PET and MRI, respectively). Interestingly, FDG-based brain

278 age prediction was slightly better than the MRI-based model (Fig. 2 and supplementary

279 table 2), implying that metabolic data may be more sensitive for tracking normal brain

280 aging trajectories. One consideration is that metabolic changes detectable on PET may

281 precede structural changes observed in $A D^{41}$, although this has not been characterized

282 in CU. Also, our FDG PET model did partially incorporate structural information since

283 the spatial normalization to template space for the FDG PET scan was performed using

284 the subject's MR images, meaning the brain-age prediction model using FDG PET has

285 the benefit of both functional and structural information. FDG PET images are also

286 affected by structural changes via partial volume effects. Alternatively, the improved

287 performance of the model using PET relative to MRI could be a consequence of

288 regional heterogeneity in age-related structural changes in the brain. ${ }^{42}$

289 Occlusion analysis shows a distinct age-specific saliency pattern according to input

290 imaging modality (Fig. 3 and supplementary Fig. 3). In the FDG-based model, a

291 transition of posterior to anterior structures with increased age was observed. The

292 posterior structures, especially the posterior cingulate cortex (PCC), contributed most in

293 younger age groups, whereas anterior structures including the frontotemporal lobes

294 were more critical in older age groups. The high contribution of PCC is consistent with

295 previously described FDG PET study demonstrating a significant correlation of glucose

296 metabolism decline in the PCC with age. ${ }^{11}$ Interestingly, amyloid deposition and

297 reduced glucose metabolism in the PCC has been implicated in early AD. ${ }^{43}$ In older 
298 adults, FDG activity in frontal regions with a peak around inferior frontal and

299 orbitofrontal, and also global activity were found to contribute the most to age prediction.

300 The decline of frontal metabolism in normal aging was consistently reported across

301 several studies. ${ }^{14,44}$ The orbitofrontal cortex is also a known region of prominent age-

302 related hypometabolism on PET. ${ }^{44}$

303

304 On the other hand, the MRI based model's saliency map demonstrated different critical

305 regions compared to the FDG PET analysis. For younger age groups, the insula was

306 identified as the most critical region. The insula has been identified as a region of gray

307 matter volume loss with normal aging. ${ }^{45}$ Additionally, the medial temporal lobe

308 structures were identified as areas with high saliency in the MRIs of younger, $30-50$

309 year old individuals, regions of previously described volume loss with aging as well as

$310 \mathrm{AD} \cdot{ }^{46}$ Preservation of brain parenchyma in the insula and medial temporal lobe of

311 younger individuals may have been a reliable feature for MRI-based age-prediction. For

312 older age groups, the cerebellomedullary cistern and the peripheral boundaries of the

313 ventricles were critical. This may reflect reliance of the age-prediction model on the

314 typical enlargement of the CSF spaces which occurs with age. ${ }^{3,4,7}$ Age-dependent

315 enlargement of the ventricles is an established phenomenon, though varies in

316 individuals. ${ }^{47}$ Interestingly, saliency maps did not show a prominent contribution of

317 cortical regions for age estimation, which we expected to find due to the typical age-

318 dependent decrease in cortical volume seen on MRI. ${ }^{45,47}$ We speculate that cortical

319 volume loss with age may be too heterogenous to serve as the most-reliable salient

320 feature for the age-prediction model. Change in white matter signal characteristics is 
321 also a well-known phenomenon of aging ${ }^{48}$ No contribution of white matter was found

322 with our occlusion analysis, which might be a consequence of white matter intensity

323 normalization performed on the MRI exams.

324 Consistent with previous findings, the estimated brain age gap of neurodegenerative

325 disease groups was larger than the CU group and significantly correlated with cognitive

326 scores. Interestingly, the estimated brain age gap is negatively correlated with

327 chronologic age for both MRI and FDG (Fig. 4) and was close to zero in older age

328 groups. This implies that normal elderly brain is indistinguishable from the diseased

329 brain at a similar older age with the DenseNet model. The brain age gap of $\mathrm{MCl}$ and $\mathrm{AD}$

330 showed a significant association with tau PET, but not amyloid PET using PiB (Fig. 7).

331 Tau is well known to be more closely related to the AD severity than $\mathrm{PiB} .{ }^{49}$ In both

332 preclinical $A D$ and $A D$ dementia, tau radiotracer uptake and cortical thickness have

333 been found to correlate with decreased cognitive task performance to a greater degree

334 than amyloid beta radiotracer uptake. ${ }^{49}$ However, the relationship between aging and

$335 \mathrm{AD}$ is complex. It has been suggested that on closer examination, differences in rates of

336 cognitive decline, structural changes, and clinical features point toward AD as a discrete

337 entity that cannot be simply described as accelerated aging process. ${ }^{40}$

338 A strong correlation was shown between FDG- and MRI-based brain age gap in the CU

339 cohort and also in the neurodegenerative disease groups (supplementary Fig. 3). This

340 result implies that the metabolic changes of normal aging, as well as disease

341 progression, are concurrent with the structural changes, with respect to factors that

342 impact the performance of the age-prediction model. The correlation between FDG- and

343 MRI-based brain age gap is mildly stronger in disease groups $(r=0.6925$ to 0.8491$)$ 
344 than in the CU cohort $(r=0.5819)$. The structural changing or atrophy in

345 neurodegenerative pathology accompanying hypometabolism, to a greater extent than

346 with normal aging, is one plausible explanation for the increased correlation in diseased

347 groups. Alternatively, brain FDG hypometabolism, which occurs in specific patterns for

348 different categories of neurodegenerative pathology ${ }^{50}$, may correlate more closely with

349 structural or volumetric changes for specific neurodegenerative disease cohorts than in

350 normal aging.

351 In FTD, frontal and anterior temporal regions showed a negative correlation with brain

352 age gap, regions with characteristic hypometabolism in $\mathrm{FTD}^{51,52}$; and a positive

353 correlation was observed in the occipital lobe, a region typically without hypometabolism

354 on FDG PET in FTD. ${ }^{51,52}$ Castelnova et al also reported that some FTD cases showed

355 occipital hypermetabolism. ${ }^{53}$ In DLB, temporal, parietal and occipital regions were

356 negatively correlated with brain age gap, regions of hypometabolism frequently

357 observed in DLB. ${ }^{51}$ Correlation of the occipital lobe and primary visual cortex in the DLB

358 group is notable because occipital/primary visual cortex hypometabolism is

359 characteristic of DLB on FDG PET from other neurodegenerative processes such as

$360 \mathrm{AD} \cdot{ }^{51,54}$ The ability of the FDG metabolic signature to distinguish DLB from $A D$ is unique

361 and an important component of the clinical utility of FDG $\mathrm{PET}^{55}$, as abnormal amyloid

362 beta PET which is a defining hallmark of $A D$, is commonly present in DLB due to the

363 phenomenon of co-occurring pathologies with advancing age.$^{54}$ The ventricle and

364 boundaries of brain parenchyma with CSF space were correlated with $\mathrm{MCl}$ and $\mathrm{AD}$ in

365 MRI. For FTD and DLB, the ventricular boundary was correlated with brain age gap,

366 although no correlation was seen at the CSF and cortical region. Periventricular borders 
367 with CSF may reflect areas of white matter volume loss and the gyral/sulcal interface

368 may reflect, which both also occur with normal aging. $3,4,7$

369 This study has some notable limitations. In the occlusion analysis, left hemispheric

370 dominance was observed in the contribution to brain age prediction, which was not

371 explainable by post-hoc analysis. The occlusion-based method has been described to

372 focus more on the most dominant regions compared to other interpretation methods. ${ }^{56}$

373 In this study, we only tested neurodegenerative pathology, without evaluating any

374 chronic systemic medical diseases and vascular diseases which may have different

375 patterns of brain aging, a limitation of this study.

377 In summary, we showed that 3D-DenseNet brain age prediction model generates

378 accurate age prediction for CU individuals, with slightly more robust performance using

379 an FDG PET input than MRI. Brain age prediction using PET imaging input, which

380 reflects metabolic function, may present a distinct assessment of brain health from the

381 structural information evaluated on MRI. The brain age gap from MRI or PET data is

382 increased in multiple types of dementia compared to CU individuals and therefore may

383 prove to be a useful composite biomarker to identify increased risk for pathology or

384 marker of disease severity.

\section{Materials and methods}

386 Dataset. A large number of participants (Table 1) ranging in age from 20 to 98 years old 387 were included $(n=2,349$, number of scans $=4,127)$ who had both MRI and FDG PET 388 from the Mayo Clinic Study of Aging (MCSA) or the Alzheimer's Disease Research 389 Center (ADRC) study (Table 1). All participants or designees provided written consent 
390 with the approval of Mayo Clinic and Olmsted Medical Center Institutional Review

391 Boards. As previously described, the Mayo Clinic Rochester ADRC is a longitudinal

392 cohort study that enrolls participants from the clinical practice at Mayo Clinic in

393 Rochester, MN. ${ }^{57}$ The MCSA is a population-based study of cognitive aging among

394 Olmsted County, MN residents. ${ }^{58}$ Enrolled participants are adjudicated to be clinically

395 normal or cognitively impaired by a consensus panel consisting of study coordinators,

396 neuropsychologists and behavioral neurologists. Methods for defining clinically

397 unimpaired, mild cognitive impairment and dementia in both of these studies conform to

398 standards in the field. ${ }^{59-61}$ For this analysis, the participants were assigned into six

399 clinical sub-groups based on clinical diagnosis following consensus criteria ${ }^{54,62}$ including

$400 \mathrm{CU}(n=1,805$, number of scans $=2,879), \mathrm{MCl}(n=480$, number of scans $=666), \mathrm{AD}(n$

$401=215$, number of scans $=372)$, FTD $(n=45$, number of scans $=69)$ and DLB $(n=86$,

402 number of scans $=141$.

403 For the CNN model training, only CU data was utilized. Some participants also

404 underwent amyloid PET scanning with PiB (number of scans=2,508) and tau PET scans

405 with flortaucipir (number of scans=608). Most participants had CDR-SB ${ }^{34}, \mathrm{STMS}^{35}$, and

406 MMSE $^{36}$ available ( $n=2,511,2,511$ and 2,464, respectively). All cognitive tests were

407 administered by experienced psychometrists and supervised by board-certified clinical

408 neuropsychologists. To examine whether the trained model presents a dataset-specific

409 bias, we also utilized ADNI dataset $(n=1,150$, number of scans $=1,622$; supplementary

410 table 1). The ADNI dataset included CU $(n=330$, number of scans $=454), \mathrm{MCl}(n=647$,

411 number of scans $=885)$ and dementia $(n=255$, number of scans $=283)$. 
413 Image processing. T1-weighted MRI scans were acquired using 3T scanners. FDG

414 PET imaging was performed with ${ }^{18} \mathrm{~F}-\mathrm{FDG}$, amyloid PET with $\mathrm{PiB}^{63}$ and tau PET with

415 Flortaucipir (AV-1451). ${ }^{64}$ FDG PET images were acquired from 30-40 minutes, PiB PET

416 from 40-60 minutes, and tau PET from 80-100 minutes after injection. CT was obtained

417 for attenuation correction. PET images were analyzed with our in-house fully automated

418 image processing pipeline ${ }^{65}$ Briefly, the PET scans were co-registered to the

419 corresponding MRI for each participant within each timepoint, and subsequently warped

420 to Mayo Clinic Adult Lifespan Template (MCALT) space 66

421 (https://www.nitrc.org/projects/mcalt/) using the warps from SPM12 Unified

422 Segmentation. ${ }^{67}$ The corresponding MRI was corrected for intensity inhomogeneity and

423 segmented using MCALT tissue priors and segmentation parameters. FDG PET SUVr

424 was calculated by dividing the median of uptake in pons and the SUVr images were

425 used for input data to the CNN model. Amyloid and tau PET SUVr were calculated by

426 dividing the median uptake in the cerebellar crus grey matter. ${ }^{37}$ A meta-ROI PiB PET

427 SUVr was derived from the average of the median SUVr in the prefrontal, orbitofrontal,

428 parietal, temporal, anterior cingulate, and posterior cingulate/precuneus regions. ${ }^{37} \mathrm{~A}$

429 meta-ROI tau PET SUVr was formed from the average of the median uptake in the

430 amygdala, entorhinal cortex, fusiform, parahippocampal and inferior temporal and

431 middle temporal gyri. ${ }^{37}$ For each MRI volume, voxels' intensities were normalized by

432 dividing a mean intensity derived from individualized white matter mask. ${ }^{68}$

434 3D-Densenet architecture and training. A modified 3D-Densenet model ${ }^{31}$ was trained 435 on FDG PET or MRI scans of cognitively unimpaired cohort (Fig. 1A). For the training, 
436 we only utilized scans of the first time point $(n=1,805)$ to avoid data leakage between

437 the training and validation/test sets. Experimental tests measuring how an overlap of 438 participants among training, validation and test sets affected the model's results were 439 performed separately (see Dataset split experiment section). A schematic of the 3D-

440 DenseNet architecture is shown in Fig 1A. The specific dimension of input data was

$441121 \times 145 \times 121$, in our applications. The output to be predicted was a single scalar

442 representing the chronological age (years). The architecture was comprised of a regular

$4433 \times 3 \times 3$ convolutional layer followed by four dense blocks and three transition blocks in 444 between them. The four dense blocks consisted of $3,6,12$, and 8 dense layers,

445 respectively (denoted above each block). Each dense layer had a $1 \times 1 \times 1$ bottleneck

446 convolutional layer followed by a $3 \times 3 \times 3$ convolution layer. The dense layers were

447 densely interconnected in a feed-forward manner within each block. The growth rate (k)

448 was 48 . The flattened output from the last global average pooling layer was then fully

449 connected with 1,457 units and was connected to the output layer.

450 The neural network was implemented using Keras with Tensorflow ${ }^{69}$ as the backend.

451 Cross-validated experiments were conducted using 5-fold validations (60\% training set, $45220 \%$ validation set and $20 \%$ test set). Mean absolute error (MAE) was used as the loss

453 function. The model was optimized using the Adam optimizer with parameters: $\beta 1=0: 9$ 454 and $\beta 2=0.99 .{ }^{70}$ The He initialization strategy was used for the weight initialization. ${ }^{71}$ The 455 training epoch was 150 . The learning rate selected for the training set was $1 \times 10^{-4}$ and 456 decreased by a factor of 2 for every ten epochs. If the validation error did not improve in 4577 epochs, the learning rate was updated. The total number of parameters were $45870,183,073$, of which $70,122,657$ were trainable parameters. We used a mini-batch size 
459 of 4 . Training and testing were performed on a Tesla P100 GPU. The source code is

460 available online (https://github.com/Neurology-AI-Program/Brain_age_prediction.git).

462 Occlusion sensitivity analysis. To facilitate interpretability, we generated brain maps

463 of the relevant features used in the age prediction model using occlusion sensitivity

464 analysis. ${ }^{72}$ The analysis was conducted within the test set. To calculate the age-specific

465 saliency map, the data were separated into seven sub-age groups based on their

466 chronological age, from 30 to 100 with 10 years interval. Within each group, the original

467 images were occluded by $11 \times 11 \times 11$ voxel areas with zero values, along a $11 \times 11 \times 11$

468 grid (Fig. 1B). Since the front and rear 12 voxels along the anterior-posterior axes do

469 not include the brain area, those were excluded from occlusion to reduce the

470 computational load. Then, age inference on the occluded images was performed

471 through our pre-trained 3D Densenet model and the performance was evaluated as

$472 \mathrm{MAE}_{\text {occlusion. }}$. The delta MAE was obtained by calculating the difference between

$473 \mathrm{MAE}_{\text {occlusion }}$ and $M A E_{\text {original }}$ acquired through the original image, and a delta MAE matrix

$474(11 \times 11 \times 11)$ was obtained by iterating occlusion for every region $(n=1,331)$. Then, the

475 delta MAE matrix was reconstructed into the original image size $(121 \times 145 \times 121)$ through

476 cubic interpolation and zero-padding for the excluded area in the occlusion, and the

477 average of the five folds was calculated. Normalization was performed by dividing the

478 entire image by the maximum value, and thus, the values of final saliency map ranged

479 from 0 to 1. 
481 Dataset split experiment. To measure how the inclusion of multiple time points per 482 participant affects brain age prediction, we tested five different data split options. The 483 main result was derived from the strictest data split option: Option 1 using only a single 484 time point per participant. Four additional options were tested: Option 2 (multiple-time 485 points per participant with overlap between training, validation, and test sets permitted);

486 Option 3 (multiple-time points per participant with overlap between training and 487 validation sets permitted); Option 4 (multiple time points for the training and validation 488 sets and a single time point for the test set; no overlap of participants amongst training, 489 validation, and test sets were permitted); and Option 5 (a single time point was used for 490 the validation and test sets; no overlap of participants among training, validation, and 491 test sets). For these five options, the validation MAE and test MAE from five-fold cross492 validations were compared (Supplementary table 3).

494 Statistical analysis. The brain age prediction accuracy was assessed by MAE and 495 Spearman correlation between predicted age and chronological age. Defining $x$ to be 496 chronological age and $y$ the predicted age, the brain age gap was calculated by $y-x$. 497 The brain age gap is known to be correlated with chronological age, which results in an 498 overestimation for younger individuals and an underestimation for older individuals ${ }^{21,38}$ 499 due to regression dilution. ${ }^{32}$ Therefore, we used the linear bias correction method 500 described $\mathrm{in}^{33}$ for age bias correction for the brain age gap. We fitted a linear regression $501 \mathrm{y}=a \mathrm{x}+b$ to the test set. Then, the corrected brain age gap was calculated by $(\mathrm{y}-b) / a-$ $502 \mathrm{x}$. The $a$ and $b$ coefficient derived from the CU group was applied to other diagnostic 503 groups in the same way for the bias correction. The corrected brain age gap of disease 
504 groups was compared with CU by a two-sample t-test. The Pearson correlation

505 coefficient was utilized to test for an association between the corrected brain age gap

506 and cognitive scores. A voxel-wise regression analysis was performed using the brain

507 age gap as a regressor to investigate which brain regions' alteration was associated

508 with brain age gap generation for each patient group. Each individual's chronological

509 age was specified as nuisance covariance. For CU participants, the same analysis was

510 performed using chronological age as a regressor. Statistical significance was corrected

511 for multiple comparisons using a false discovery rate $(F D R)^{73}$ with a cluster size of at

512 least 100 adjacent voxels. An association of corrected brain age gap with meta-ROI PiB

513 PET SUVr and meta-ROI tau PET SUVr was assessed by Pearson correlation.

\section{Acknowledgement}

516 We gratefully acknowledge the support of NVIDIA Corporation with the donation of the

517 Tesla P100 GPU used for this research.

518 This work was funded in part by NIH grants P30 AG62677-2 (D.J.), R01 AG011378

519 (C.J.), R01 AG041851 (C.J.), P50 AG016574 (R.P.), U01 AG06786 (R.P.), and by the

520 Robert Wood Johnson Foundation, The Elsie and Marvin Dekelboum Family

521 Foundation, The Edson Family Foundation, The Liston Family Foundation, the Robert

$522 \mathrm{H}$. and Clarice Smith and Abigail van Buren Alzheimer's Disease Research Program,

523 The GHR Foundation, Foundation Dr. Corinne Schuler (Geneva, Switzerland), Race

524 Against Dementia, and the Mayo Foundation. 
527 Conceptualization: J.L., HK. M., and D.T.J. Software: J.L. and L.R.B. Preprocessing:

528 M.L.S. Methodology: J.L., H.K.M., M.L.S., E.S.L., H.B., and C.G.S. Writing—original

529 draft: J.L., B.J.B., H.K.M., V.J.L. and D.T.J. Writing- revisions: All authors. Supervision:

530 V.J.L., C.R.J., and D.T.J. All authors have given final approval of this version of the

531 article.

532

533 Competing interests

534 The authors report no competing interests.

535

\section{References}

5371 López-Otín, C., Blasco, M. A., Partridge, L., Serrano, M. \& Kroemer, G. The hallmarks 538 of aging. Cell 153, 1194-1217 (2013).

5392 Harman, D. Aging: overview. Annals of the New York Academy of Sciences 928, 1-21

$540 \quad$ (2001).

5413 Courchesne, E. et al. Normal brain development and aging: quantitative analysis at in

$542 \quad$ vivo MR imaging in healthy volunteers. Radiology 216, 672-682 (2000).

5434 Good, C. D. et al. A voxel-based morphometric study of ageing in 465 normal adult

$544 \quad$ human brains. Neuroimage 14, 21-36 (2001).

5455 Sowell, E. R. et al. Mapping cortical change across the human life span. Nature

546 neuroscience 6, 309-315 (2003).

5476 Lemaitre, H. et al. Normal age-related brain morphometric changes: nonuniformity across cortical thickness, surface area and gray matter volume? Neurobiology of aging 33, 617. e611-617. e619 (2012).

7 Raz, N. \& Rodrigue, K. M. Differential aging of the brain: patterns, cognitive correlates

5528 Walhovd, K. B. et al. Effects of age on volumes of cortex, white matter and subcortical structures. Neurobiology of aging 26, 1261-1270 (2005). Goyal, M. S. et al. Loss of brain aerobic glycolysis in normal human aging. Cell

9 Goyal, M. S. et al. Loss of brain aerob

10 Goyal, M. S., Hawrylycz, M., Miller, J. A., Snyder, A. Z. \& Raichle, M. E. Aerobic glycolysis in the human brain is associated with development and neotenous gene expression. Cell metabolism 19, 49-57 (2014). analysis for multivariate $3 \mathrm{D}$ voxel-based mapping of brain functional imaging data sets as applied to FDG-PET and normal aging. Human brain mapping 18, 13-21 (2003). 
56212 Knopman, D. S. et al. 18F-fluorodeoxyglucose positron emission tomography, aging, and 563 apolipoprotein E genotype in cognitively normal persons. Neurobiology of aging 35, 564 2096-2106 (2014).

56513 De Leon, M. et al. Prediction of cognitive decline in normal elderly subjects with 2-[18F] fluoro-2-deoxy-D-glucose/positron-emission tomography (FDG/PET). Proceedings of the National Academy of Sciences 98, 10966-10971 (2001).

14 De Santi, S. et al. Age-related changes in brain: II. Positron emission tomography of frontal and temporal lobe glucose metabolism in normal subjects. Psychiatric Quarterly 66, 357-370 (1995).

15 Bonte, S. et al. Healthy brain ageing assessed with 18F-FDG PET and age-dependent recovery factors after partial volume effect correction. European journal of nuclear medicine and molecular imaging 44, 838-849 (2017).

16 Shen, X., Liu, H., Hu, Z., Hu, H. \& Shi, P. The relationship between cerebral glucose metabolism and age: report of a large brain PET data set. PloS one 7, e51517 (2012).

17 Petit-Taboue, M., Landeau, B., Desson, J., Desgranges, B. \& Baron, J. Effects of healthy aging on the regional cerebral metabolic rate of glucose assessed with statistical parametric mapping. Neuroimage 7, 176-184 (1998).

18 Goyal, M. S. et al. Persistent metabolic youth in the aging female brain. Proceedings of the National Academy of Sciences 116, 3251-3255 (2019).

19 Cole, J. H. \& Franke, K. Predicting age using neuroimaging: innovative brain ageing biomarkers. Trends in neurosciences 40, 681-690 (2017).

20 Cole, J. H. Multimodality neuroimaging brain-age in UK biobank: relationship to biomedical, lifestyle, and cognitive factors. Neurobiology of aging 92, 34-42 (2020).

21 Bashyam, V. M. et al. MRI signatures of brain age and disease over the lifespan based on a deep brain network and 14468 individuals worldwide. Brain 143, 2312-2324 (2020).

22 Abrol, A. et al. Deep learning encodes robust discriminative neuroimaging representations to outperform standard machine learning. Nature communications 12, 117 (2021).

23 Cole, J. H. et al. Predicting brain age with deep learning from raw imaging data results in a reliable and heritable biomarker. NeuroImage 163, 115-124 (2017).

24 Levakov, G., Rosenthal, G., Shelef, I., Raviv, T. R. \& Avidan, G. From a deep learning model back to the brain-Identifying regional predictors and their relation to aging. Human brain mapping 41, 3235-3252 (2020).

25 Jónsson, B. A. et al. Brain age prediction using deep learning uncovers associated sequence variants. Nature communications 10, 1-10 (2019).

26 Cole, J. H. et al. Brain age predicts mortality. Molecular psychiatry 23, 1385-1392 (2018).

27 Gaser, C. et al. BrainAGE in mild cognitive impaired patients: predicting the conversion to Alzheimer's disease. PloS one 8, e67346 (2013).

28 Habes, M. et al. Advanced brain aging: relationship with epidemiologic and genetic risk factors, and overlap with Alzheimer disease atrophy patterns. Translational psychiatry $\mathbf{6}$, e775-e775 (2016).

29 Jones, D. T. et al. Age-related changes in the default mode network are more advanced in Alzheimer disease. Neurology 77, 1524-1531 (2011). 
60630 Lorenzi, M., Pennec, X., Frisoni, G. B., Ayache, N. \& Initiative, A. s. D. N.

607 Disentangling normal aging from Alzheimer's disease in structural magnetic resonance

$608 \quad$ images. Neurobiology of aging 36, S42-S52 (2015).

60931 Huang, G., Liu, Z., Van Der Maaten, L. \& Weinberger, K. Q. in Proceedings of the IEEE

$610 \quad$ conference on computer vision and pattern recognition. 4700-4708.

61132 MacMahon, S. et al. Blood pressure, stroke, and coronary heart disease: part 1, prolonged

612

613

614 differences in blood pressure: prospective observational studies corrected for the regression dilution bias. The Lancet 335, 765-774 (1990).

615

33 Smith, S. M., Vidaurre, D., Alfaro-Almagro, F., Nichols, T. E. \& Miller, K. L. Estimation of brain age delta from brain imaging. NeuroImage 200, 528-539 (2019).

34 Morris, J. C. Clinical dementia rating: a reliable and valid diagnostic and staging measure for dementia of the Alzheimer type. International psychogeriatrics 9, 173-176 (1997). Kokmen, E., Smith, G. E., Petersen, R. C., Tangalos, E. \& Ivnik, R. C. The short test of mental status: correlations with standardized psychometric testing. Archives of neurology 48, 725-728 (1991).

36 Folstein, M. F., Folstein, S. E. \& McHugh, P. R. "Mini-mental state": a practical method for grading the cognitive state of patients for the clinician. Journal of psychiatric research 12, 189-198 (1975).

40 Toepper, M. Dissociating normal aging from Alzheimer's disease: A view from cognitive Alzheimer's disease. Alzheimer's \& Dementia 13, 205-216 (2017).

\section{neuroscience. Journal of Alzheimer's disease 57, 331-352 (2017).}

41 Chételat, G. et al. Direct voxel-based comparison between grey

42 Salat, D. H. et al. Thinning of the cerebral cortex in aging. Cerebral cortex 14, 721-730 (2004).

43 Buckner, R. L. et al. Molecular, structural, and functional characterization of Alzheimer's disease: evidence for a relationship between default activity, amyloid, and memory. Journal of neuroscience 25, 7709-7717 (2005).

44 Curiati, P. et al. Age-Related Metabolic Profiles in Cognitively Healthy Elders: Results from a Voxel-Based [18F] Fluorodeoxyglucose-Positron-Emission Tomography Study with Partial Volume Effects Correction. American journal of neuroradiology 32, 560-565 (2011).

45 Long, X. et al. Healthy aging: an automatic analysis of global and regional morphological alterations of human brain. Academic radiology 19, 785-793 (2012).

46 Jack, C. R. et al. Rate of medial temporal lobe atrophy in typical aging and Alzheimer's disease. Neurology 51, 993-999 (1998).

47 Davis, P. C., Mirra, S. S. \& Alazraki, N. The brain in older persons with and without dementia: findings on MR, PET, and SPECT images. AJR. American journal of roentgenology 162, 1267-1278 (1994). 
65148 Habes, M. et al. White matter hyperintensities and imaging patterns of brain ageing in the general population. Brain 139, 1164-1179 (2016). Ossenkoppele, R. et al. Associations between tau, A $\beta$, and cortical thickness with cognition in Alzheimer disease. Neurology 92, e601-e612 (2019).

50 Shivamurthy, V. K., Tahari, A. K., Marcus, C. \& Subramaniam, R. M. Brain FDG PET and the diagnosis of dementia. American Journal of Roentgenology 204, W76-W85 (2015).

51 Brown, R. K., Bohnen, N. I., Wong, K. K., Minoshima, S. \& Frey, K. A. Brain PET in

52 Kanda, T. et al. Comparison of grey matter and metabolic reductions in frontotemporal dementia using FDG-PET and voxel-based morphometric MR studies. European journal of nuclear medicine and molecular imaging 35, 2227-2234 (2008).

53 Castelnovo, V. et al. Heterogeneous brain FDG-PET metabolic patterns in patients with C9orf72 mutation. Neurological Sciences 40, 515-521 (2019).

54 McKeith, I. G. et al. Diagnosis and management of dementia with Lewy bodies: Fourth consensus report of the DLB Consortium. Neurology 89, 88-100 (2017).

55 Graff-Radford, J. et al. 18F-fluorodeoxyglucose positron emission tomography in dementia with Lewy bodies. Brain communications 2, fcaa040 (2020).

56 Rieke, J., Eitel, F., Weygandt, M., Haynes, J.-D. \& Ritter, K. in Understanding and Interpreting Machine Learning in Medical Image Computing Applications 24-31 (Springer, 2018).

57 Jones, D. T. et al. Tau, amyloid, and cascading network failure across the Alzheimer's disease spectrum. Cortex 97, 143-159 (2017).

58 Roberts, R. O. et al. The Mayo Clinic Study of Aging: design and sampling, participation, baseline measures and sample characteristics. Neuroepidemiology 30, 5869 (2008).

59 Albert, M. S. et al. The diagnosis of mild cognitive impairment due to Alzheimer's disease: recommendations from the National Institute on Aging-Alzheimer's Association workgroups on diagnostic guidelines for Alzheimer's disease. Alzheimer's \& dementia 7, 270-279 (2011).

60 McKhann, G. M. et al. The diagnosis of dementia due to Alzheimer's disease: recommendations from the National Institute on Aging-Alzheimer's Association workgroups on diagnostic guidelines for Alzheimer's disease. Alzheimer's \& dementia 7, 263-269 (2011).

61 Petersen, R. C. Mild cognitive impairment as a diagnostic entity. Journal of internal medicine 256, 183-194 (2004).

62 Neary, D. et al. Frontotemporal lobar degeneration: a consensus on clinical diagnostic criteria. Neurology 51, 1546-1554 (1998).

63 Klunk, W. E. et al. Imaging brain amyloid in Alzheimer's disease with Pittsburgh 694 Compound-B. Annals of Neurology: Official Journal of the American Neurological Association and the Child Neurology Society 55, 306-319 (2004). 
69565 Schwarz, C. G. et al. A comparison of partial volume correction techniques for 696 measuring change in serial amyloid PET SUVR. Journal of Alzheimer's Disease 67, 181$697 \quad 195(2019)$.

69866 Schwarz, C. et al. in Alzheimer's Association International Conference.[Google 699 Scholar].

70067 Ashburner, J. \& Friston, K. J. Unified segmentation. Neuroimage 26, 839-851 (2005).

70168 Shinohara, R. T. et al. Statistical normalization techniques for magnetic resonance 702 imaging. NeuroImage: Clinical 6, 9-19 (2014).

70369 Abadi, M. et al. in 12th \{USENIX\} symposium on operating systems design and 704 implementation (\{OSDI\} 16). 265-283.

$70570 \quad$ Kingma, D. P. \& Ba, J. Adam: A method for stochastic optimization. arXiv preprint 706 arXiv:1412.6980 (2014).

70771 He, K., Zhang, X., Ren, S. \& Sun, J. in Proceedings of the IEEE international conference 708 on computer vision. 1026-1034.

70972 Zeiler, M. D. \& Fergus, R. in European conference on computer vision. 818-833 $710 \quad$ (Springer).

71173 Genovese, C. R., Lazar, N. A. \& Nichols, T. Thresholding of statistical maps in 
Figures and Tables.

Figure1. 3D Densenet architecture for age prediction and layout of occlusion analysis.

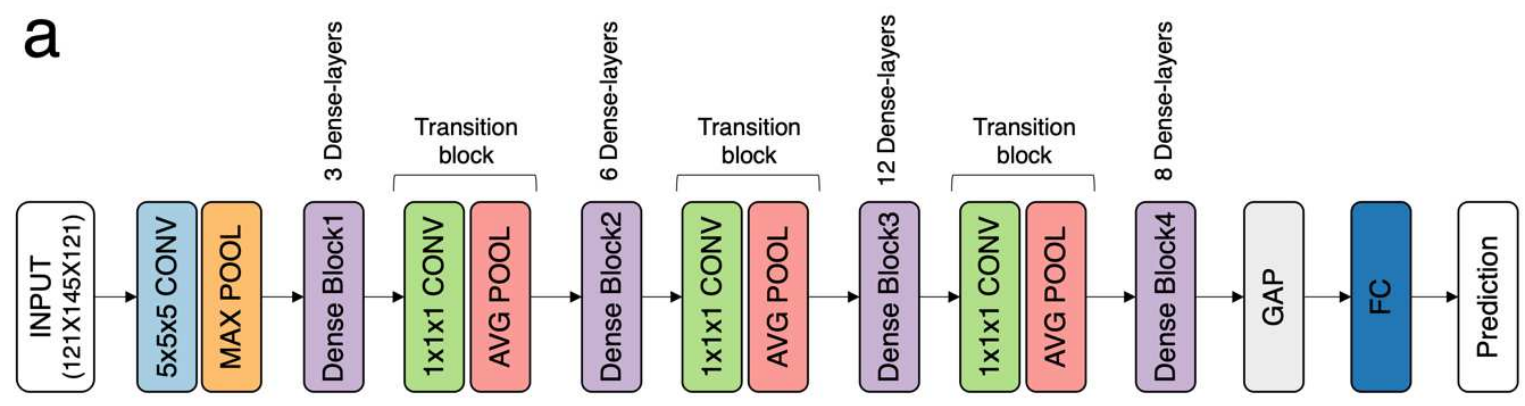

D For age subgroup

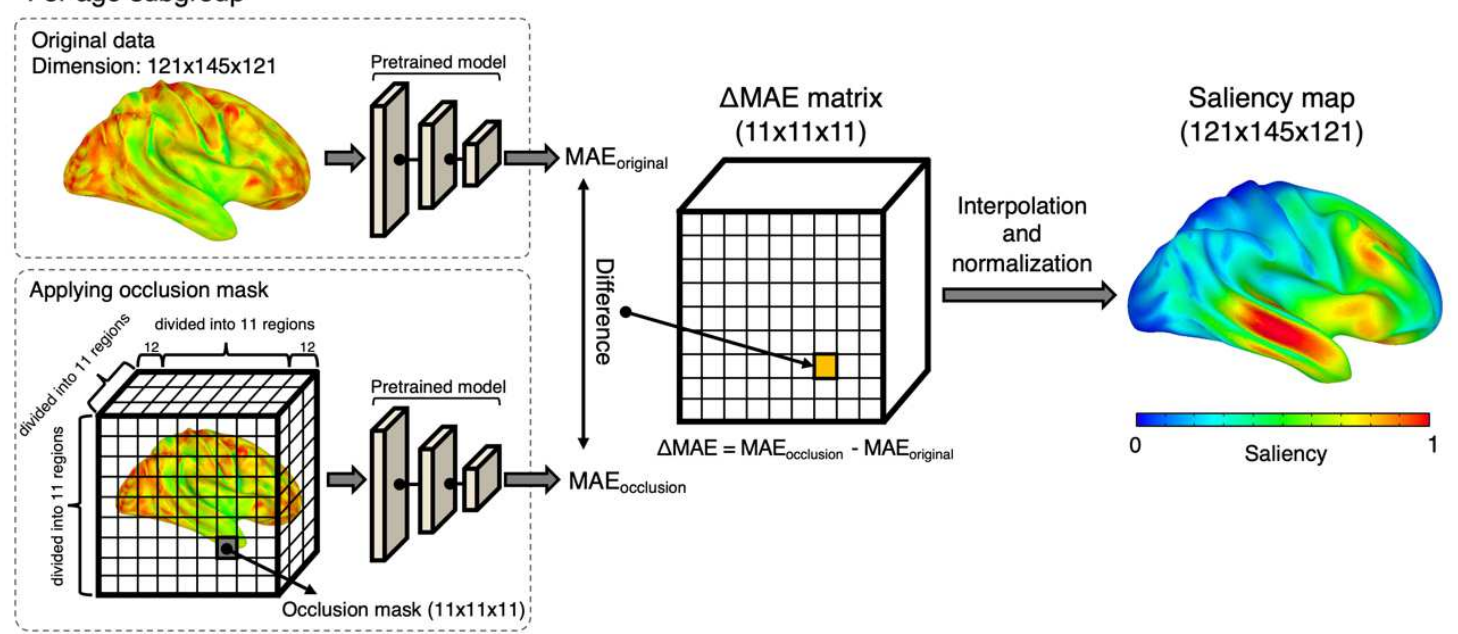

Figure1. 3D Densenet architecture for age prediction and layout of occlusion

analysis. a, The detailed architecture of the 3D Densenet used for age prediction.

CONV = convolutional layer, $\mathrm{MAX} \mathrm{POOL}=\max$ pooling layer, $\mathrm{AVG} \mathrm{POOL}=$ average pooling layer, GAP = global average pooling layer, FC = fully connected layer. $\mathbf{b}$, Illustration of the framework for occlusion analysis. 
Figure 2. Brain age predictions on CU participants.

a

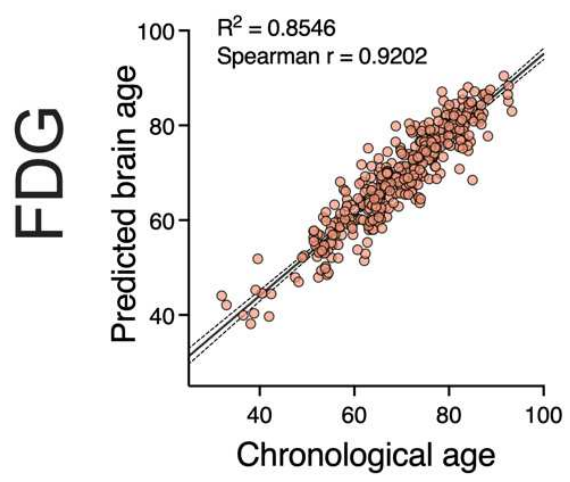

d

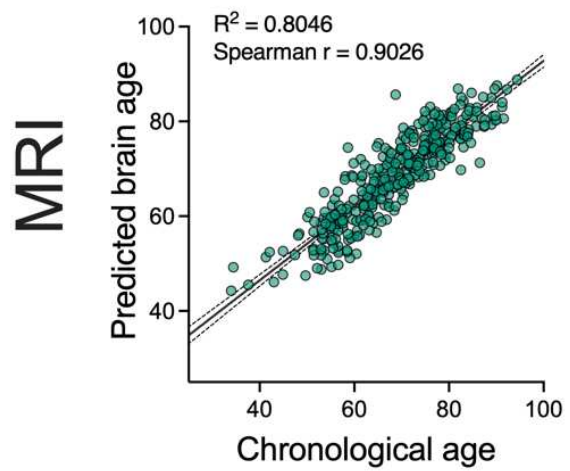

b

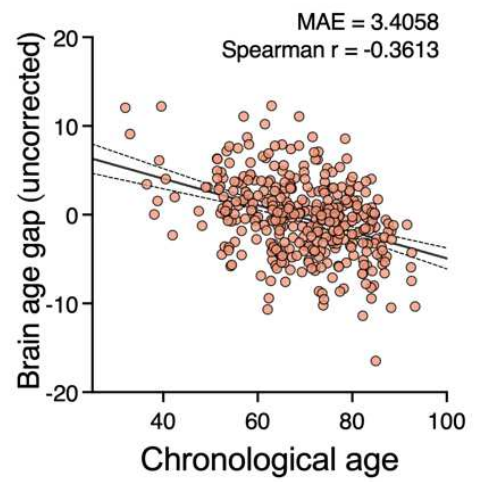

e

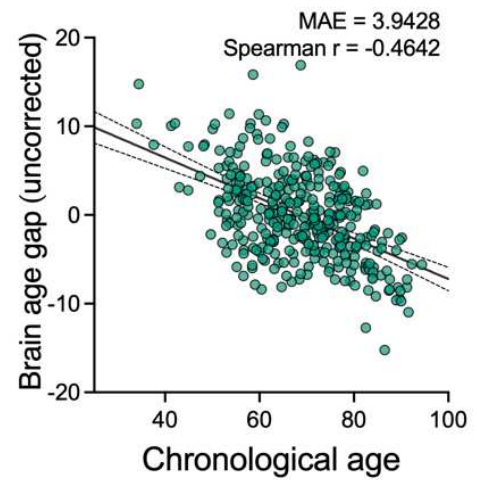

C

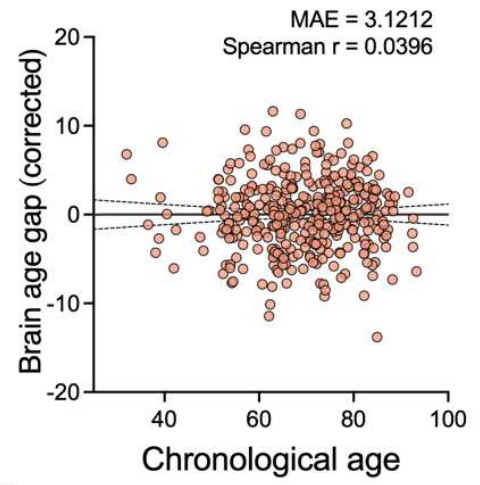

$\mathrm{f}$

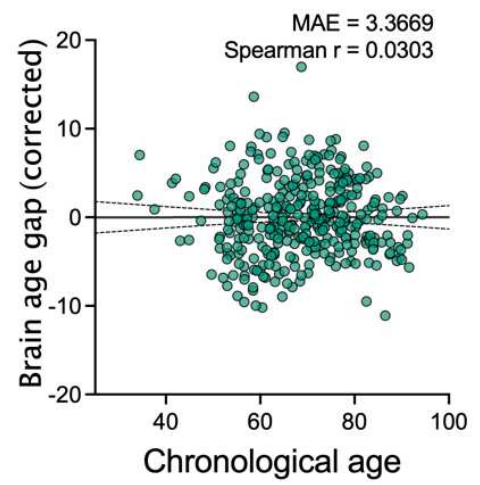

Figure 2. Brain age predictions on CU participants. (a-c) FDG based brain age

prediction result for the test set of the representative fold. a, A regression plot showing

732 chronological age vs. predicted brain age. b, The uncorrected brain age gap. c, The

733 brain age gap after bias correction. (d-f) MRI-based brain age prediction result for the

734 test set of the representative fold. $\mathbf{d}$, A regression plot showing chronological age vs.

735 predicted brain age. e, The uncorrected brain age gap. $\mathbf{f}$, The brain age gap after bias

736 correction. The black solid line and dotted lines in each figure represent a regression

737 line and its $95 \%$ confidence bands, respectively. 
739 Figure 3. The visualization of model activation shown on coronal slices.

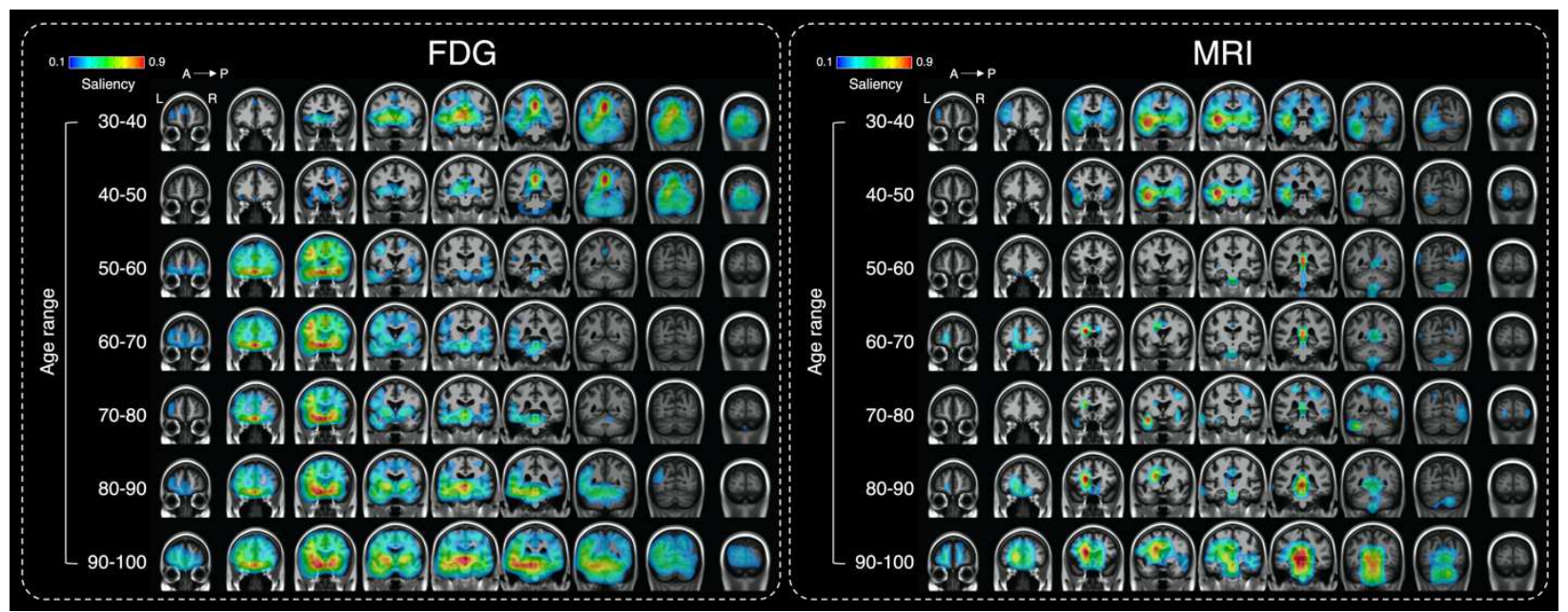

744 maps were computed using occlusion sensitivity analysis for each age range group.

745 Higher activation represents the importance of a region in brain age estimation. A left

746 panel shows the saliency maps for the FDG-based model and a right panel shows the

747 saliency maps for MRI-based model. 
Figure 4. Regression plots of a corrected brain age gap as a function of chronological age for clinical diagnostic groups.
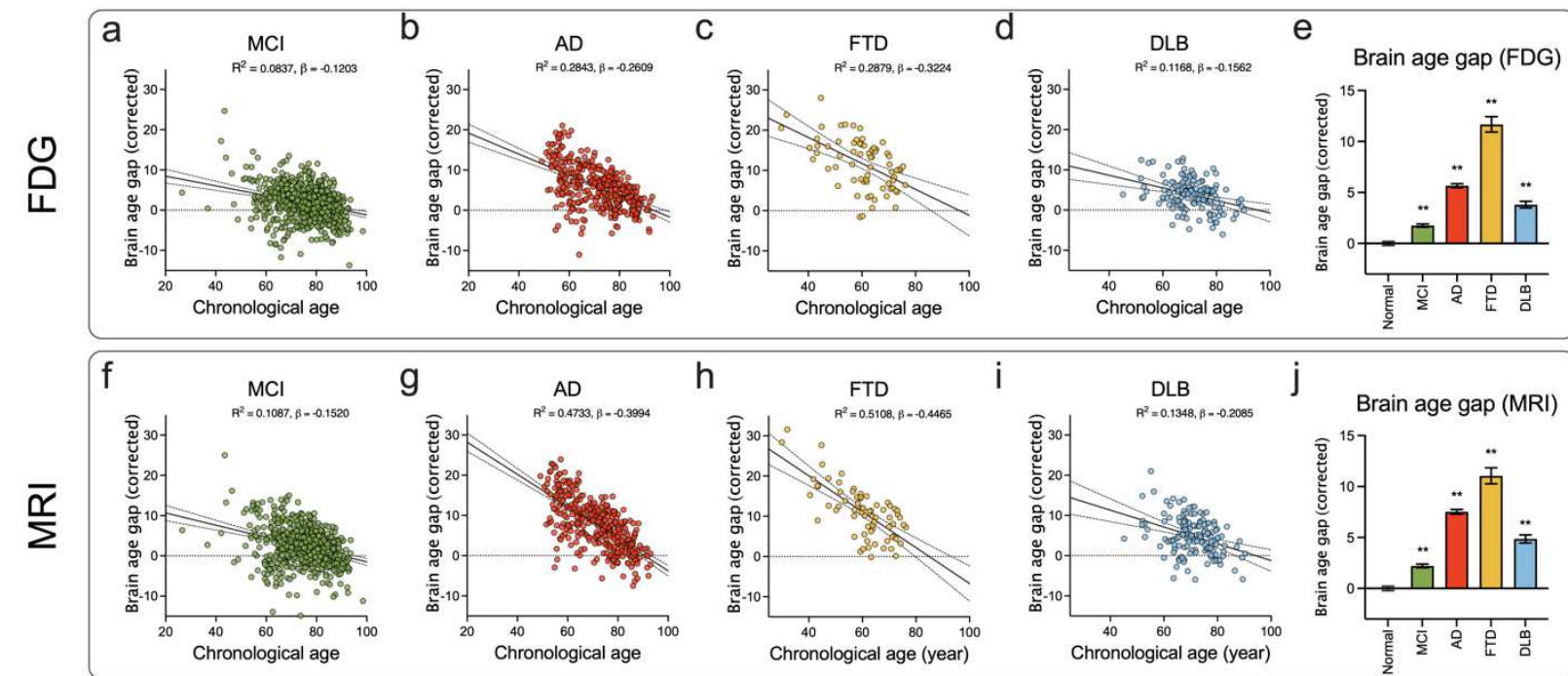

Figure 4. Regression plots of a corrected brain age gap as a function of

chronological age for clinical diagnostic groups. (a-d) FDG-based brain age gap

757 estimation for $\mathrm{MCl}, \mathrm{AD}, \mathrm{FTD}$ and DLB, respectively. e, Mean of corrected brain age gap

758 for each diagnostic group. Error bars indicate the standard error of the mean. ${ }^{* *} p<0.001$

759 comparison with CU, two-sample t-test. (f-i) MRI-based brain age gap estimation for

$760 \mathrm{MCl}, \mathrm{AD}, \mathrm{FTD}$ and DLB, respectively. j, Mean of corrected brain age gap for each

761 clinical diagnosis group. Error bars indicate the standard error of the mean. ${ }^{* *} p<0.001$

762 comparison with CU, two-sample t-test. 
764 Figure 5. Voxel-wise linear regression analysis of brain age gap.

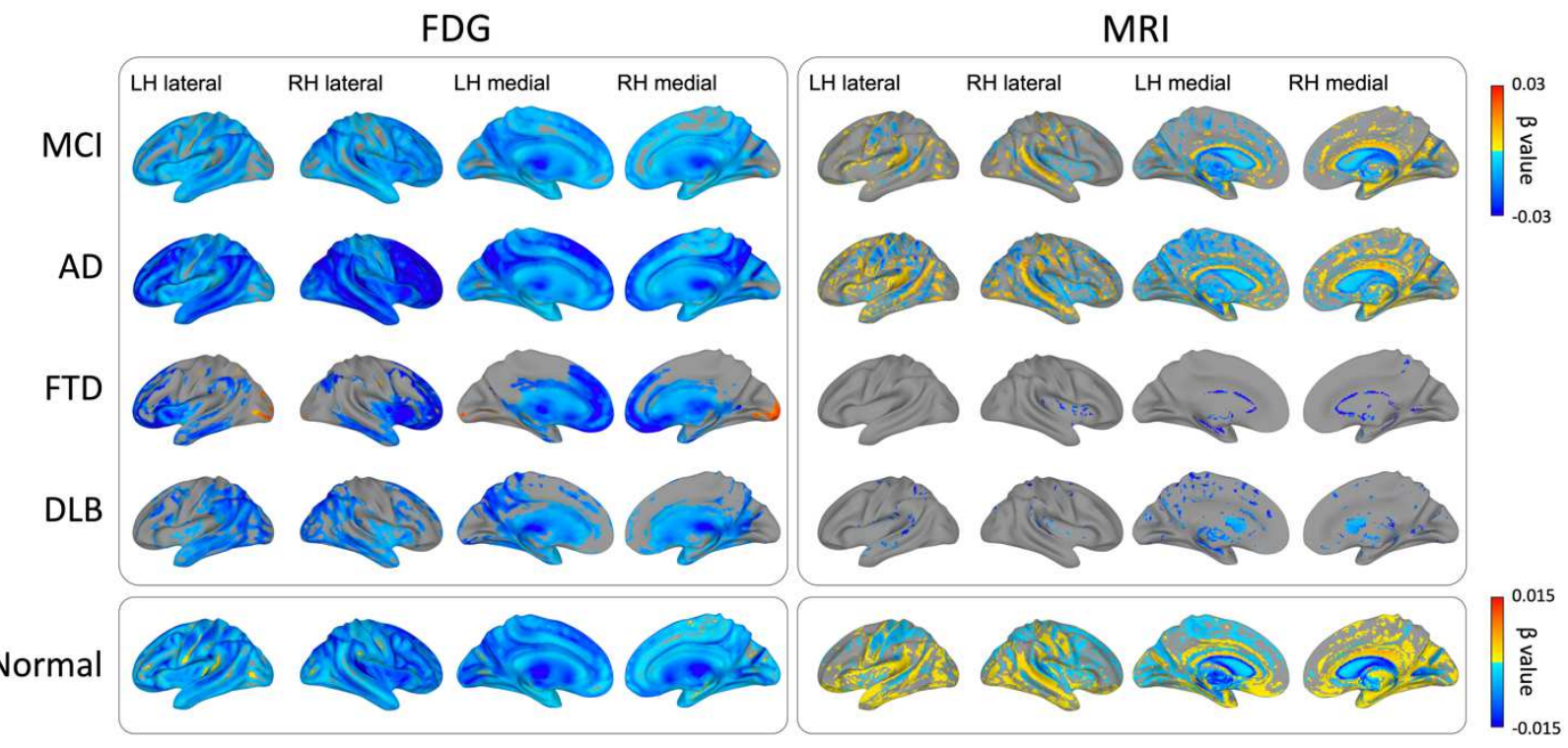

767 Figure 5. Voxel-wise linear regression analysis of brain age gap. Clinical diagnosis

768 group (MCl, AD, FTD and DLB)-specific results from voxel-wise whole-brain linear

769 regression examining the brain age gap-related change (FDR corrected, $q<0.01$ ). The

770 chronological age was specified as nuisance covariance. For CU (bottom row), voxel-

771 wise linear regression analysis was performed using the chronological age as a

772 regressor to show the age-related change. A left panel shows the results for the FDG-

773 based model and a right panel shows the results for the MRI-based model. 
Figure 6. Association of a brain age gap with cognitive scores.
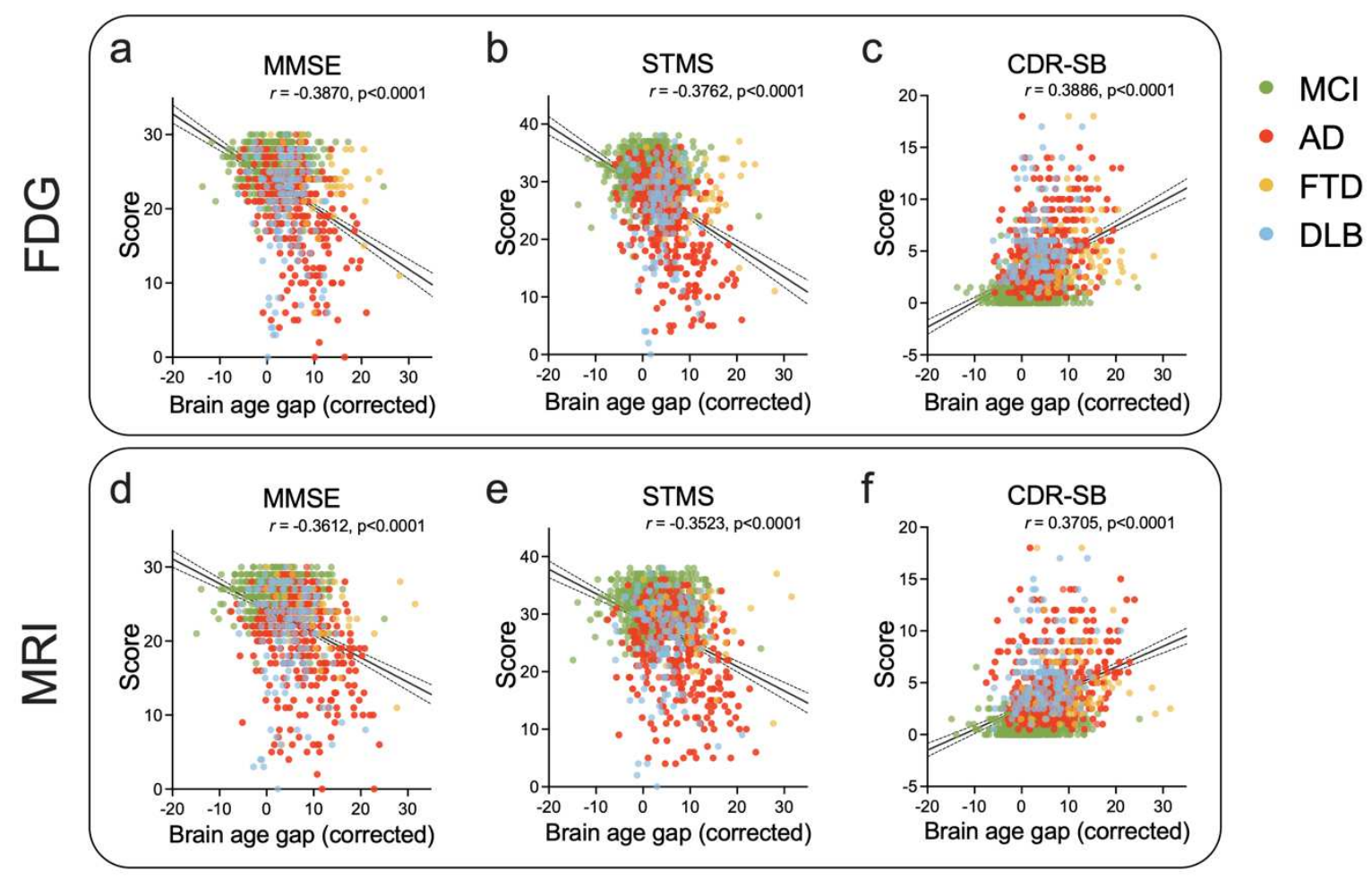

776

777

778

779

780

781 and CDR-SB, respectively.
Figure 6. Association of a brain age gap with cognitive scores. (a-c) Scatter plots of FDG model-based brain age gap with Mini-Mental State Examinations (MMSE), Short Test of Mental Status (STMS) and Clinical Dementia Rating Sum of boxes (CDR-SB), respectively. (d-f) Scatter plots of MRI model-based brain age gap with MMSE, STMS 
Figure 7. Association of brain age gap with meta-ROI PiB- and Tau PET SUVr.
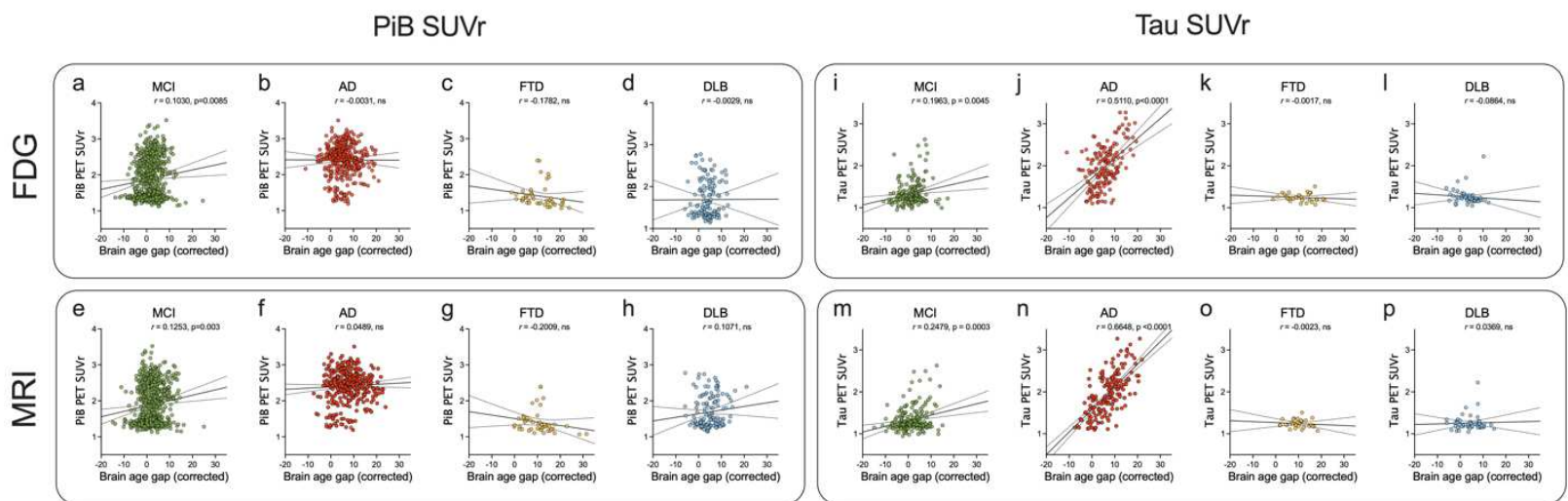

Figure 7. Association of brain age gap with meta-ROI PiB- and Tau PET SUVr. (a-

d) Scatter plots show the relationship between FDG-based brain age gap with metaROI PiB PET SUVr for MCI, AD, FTD and DLB, respectively. (e-h) Scatter plots show

790 the relationship between MRI-based brain age gap with meta-ROI PiB SUVr for MCl,

791 AD, FTD and DLB, respectively. (i-I) Scatter plots show the relationship between FDG-

792 based brain age gap with meta-ROI Tau PET SUVr for MCI, AD, FTD and DLB,

793 respectively. (m-p) Scatter plots show the relationship between MRI-based brain age

794 gap with meta-ROI Tau PET SUVr for MCI, AD, FTD and DLB, respectively. The black

795 solid line and dotted lines in each figure represent a regression line and its $95 \%$

796 confidence bands, respectively. $r$ indicates Pearson's correlation coefficient. 


\section{Table 1. Demographics of Mayo dataset}

\begin{tabular}{|c|c|c|c|c|c|}
\hline \multirow{2}{*}{ Characteristic } & \multicolumn{5}{|c|}{ Clinical Diagnosis } \\
\hline & Normal & MCI & $A D$ & FTD & DLB \\
\hline $\mathrm{N}$ & 1,805 & 480 & 215 & 45 & 86 \\
\hline \multicolumn{6}{|l|}{ Total time points, $\mathrm{n}(\%)$} \\
\hline 1 & $973(53.91)$ & $190(39.58)$ & $80(37.21)$ & $19(42.22)$ & $44(51.16)$ \\
\hline 2 & $503(27.87)$ & $130(27.08)$ & $72(33.49)$ & $10(22.22)$ & $15(17.44)$ \\
\hline 3 & $243(13.46)$ & $86(17.92)$ & $31(14.42)$ & $8(17.78)$ & $10(11.63)$ \\
\hline $4+$ & $86(4.76)$ & $74(15.42)$ & $32(14.88)$ & $8(17.78)$ & $17(19.76)$ \\
\hline \multicolumn{6}{|l|}{ Age, years } \\
\hline Median (IQR) & $72(6279)$ & $77(70$ 83) & $\begin{array}{l}74(64 \\
79.75)\end{array}$ & $\begin{array}{l}63(55 \\
70.25)\end{array}$ & $71(6677)$ \\
\hline Min Max & 3097 & 2698 & 4992 & 3176 & 4590 \\
\hline Male sex, $\mathrm{n}(\%)$ & $952(52.74)$ & $319(66.46)$ & $117(54.42)$ & $26(57.78)$ & $74(86.05)$ \\
\hline Education, years, median (IQR) & $15(1317)$ & $14(1216)$ & $\begin{array}{l}16(12 \\
17.75)\end{array}$ & $\begin{array}{l}16(13 \\
17.25)\end{array}$ & $15.5(1318)$ \\
\hline $\begin{array}{l}\text { Clinical Dementia Rating Scale- } \\
\text { Sum of Boxes, median (IQR) }\end{array}$ & $0\left(\begin{array}{llll}0 & 0\end{array}\right)$ & $\begin{array}{l}0.5(0.5 \\
0.5)\end{array}$ & $1(0.51)$ & $1(0.51)$ & $1(0.51)$ \\
\hline $\begin{array}{l}\text { Mini-Mental State Examinations, } \\
\text { median (IQR) }\end{array}$ & 29 (28 29) & $27(2428)$ & $21(17$ 24) & $24(2126)$ & $\begin{array}{l}23(17 \\
25.25)\end{array}$ \\
\hline $\begin{array}{l}\text { Short Test of Mental Status, } \\
\text { median (IQR) }\end{array}$ & 36 (34 37) & 32 (29 34) & 25 (19 29) & 28 (25 31.5) & $27(2230)$ \\
\hline
\end{tabular}

799

800 
Supplementary figure 1. Brain age predictions on the ADNI dataset.

a

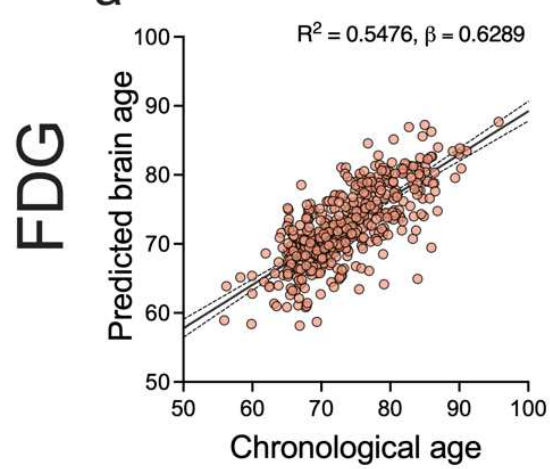

d

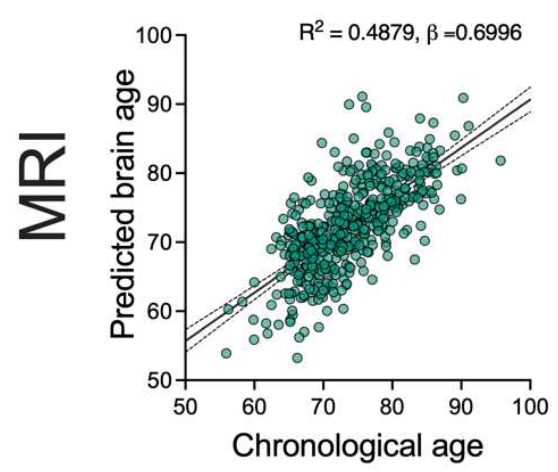

b

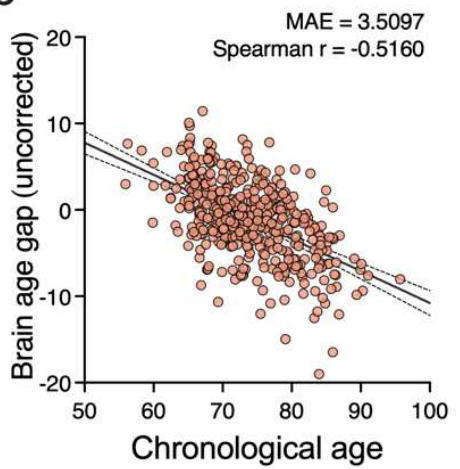

e

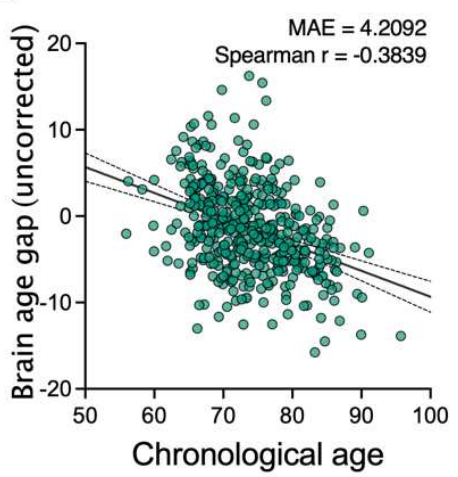

C

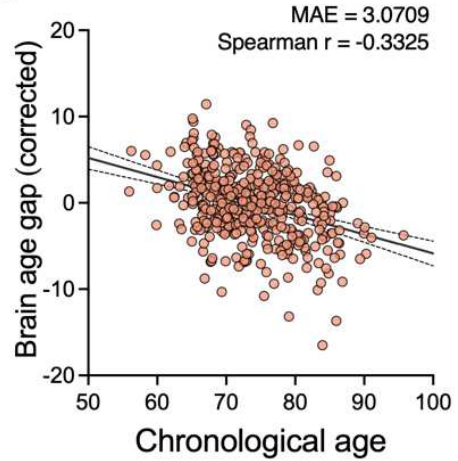

$f$

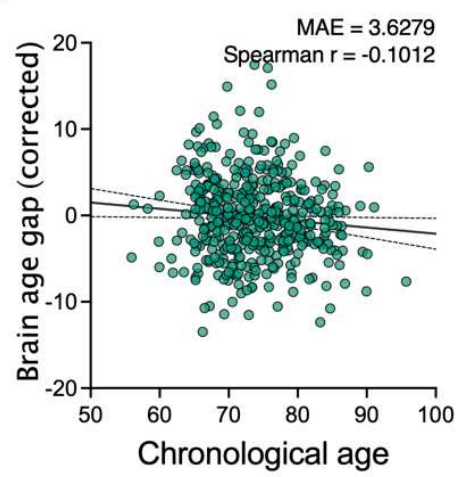

802

803

804

805

806

807

808

809

810 811 lines in each figure represent a regression line and its $95 \%$ confidence bands, 812 respectively.

Supplementary figure 1. Brain age predictions on the ADNI dataset. 3D Densenet model trained on the Mayo dataset was applied to the ADNI data. (a-c) FDG based brain age prediction result for the test set. a, A regression plot showing chronological age vs. predicted brain age. $\mathbf{b}$, The uncorrected brain age gap. $\mathbf{c}$, The brain age gap after bias correction. (d-f) MRI-based brain age prediction result for the test set. $\mathbf{d}, \mathrm{A}$ regression plot showing chronological age vs. predicted brain age. e, The uncorrected brain age gap. $\mathbf{f}$, The brain age gap after bias correction. The black solid line and dotted 

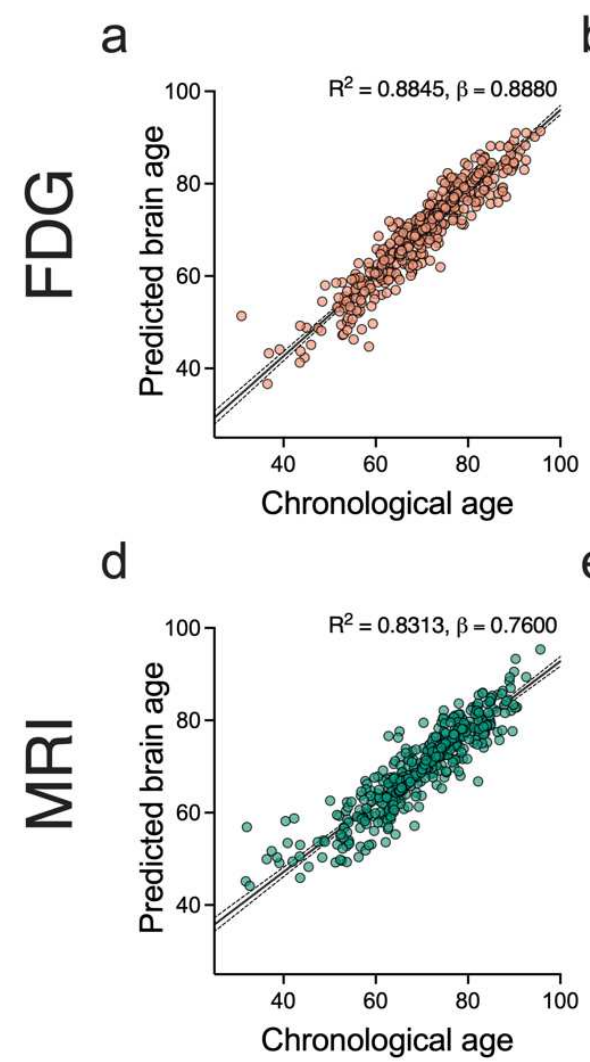

b

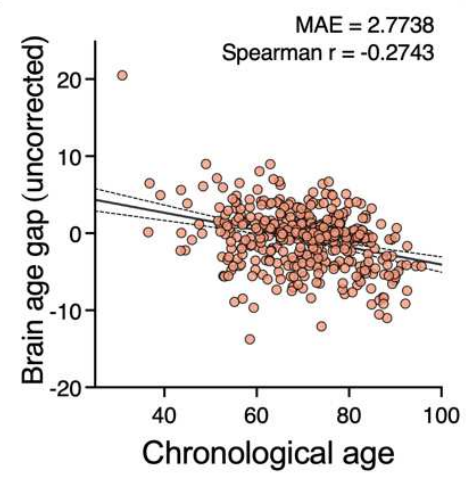

e

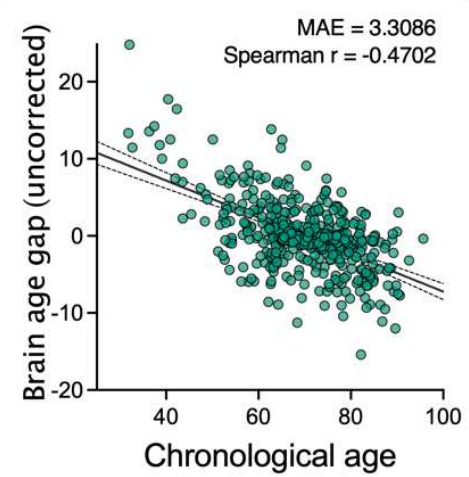

C
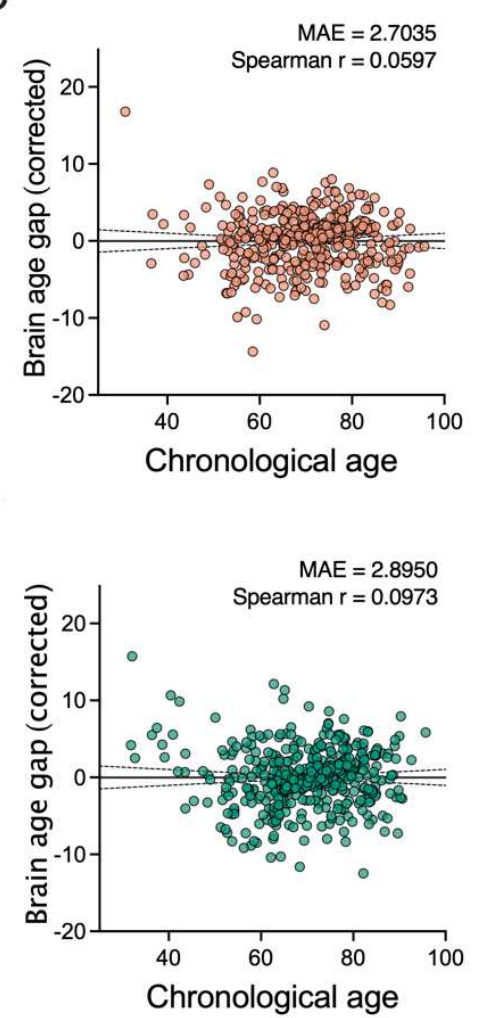

817

821 plot showing chronological age vs. predicted brain age. $\mathbf{b}$, The uncorrected brain age 822 gap. c, The brain age gap after bias correction. (d-f) MRI-based brain age prediction

823 result for the test set. $\mathbf{d}$, A regression plot showing chronological age vs. predicted brain

824 age. e, The uncorrected brain age gap. $\mathbf{f}$, The brain age gap after bias correction. The black solid line and dotted lines in each figure represent a regression line and its $95 \%$

826 confidence bands, respectively.

\section{Supplementary figure 2. Brain age predictions on the Mayo + ADNI dataset.}

Prediction performance of 3D Densenet model trained on the Mayo and ADNI dataset together. (a-c) FDG based brain age prediction result for the test set. a, A regression 
828 Supplementary figure 3. Regional mean saliency.
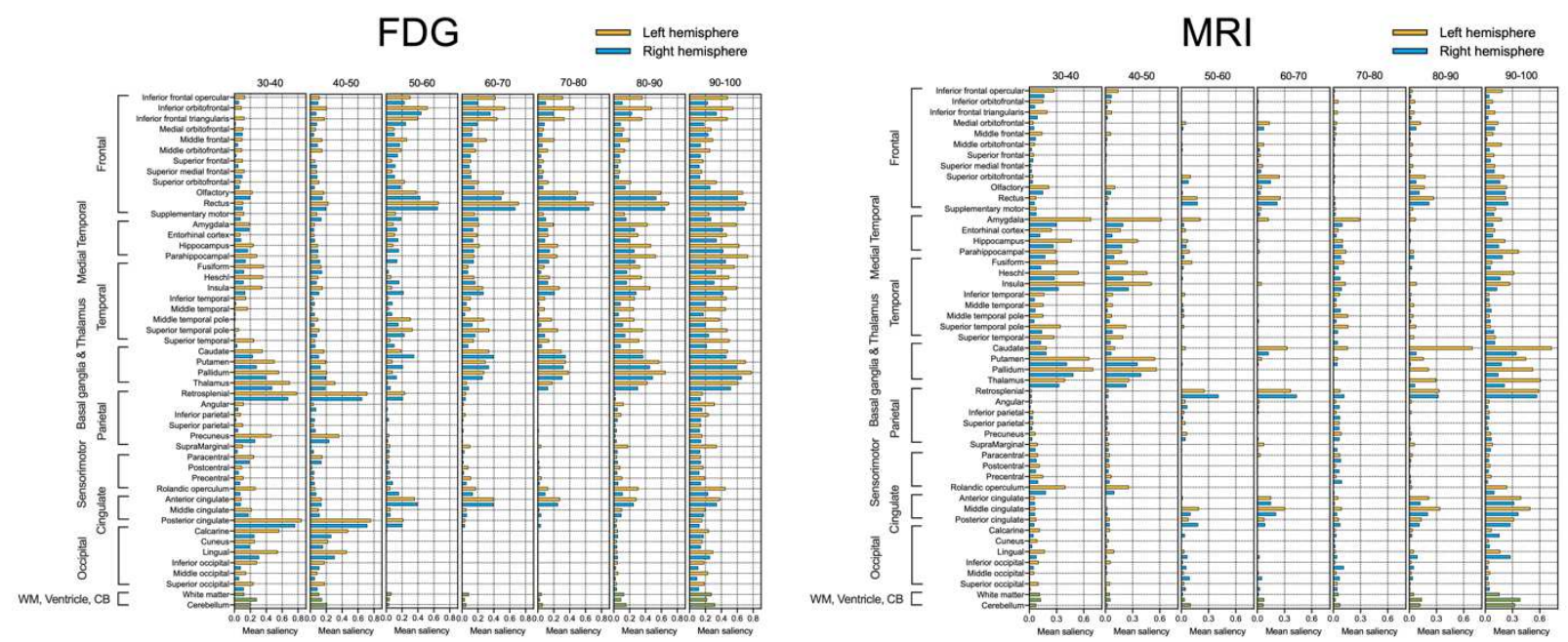

829

830

831

Supplementary figure 3. Regional mean saliency. After calculating the saliency map

832 from occlusion analysis, mean saliency value was calculated for each ROI. Yellow-

833 colored bars indicate the left hemisphere and blue-colored bars indicate the right

834 hemisphere.

835 
836 Supplementary figure 4. Relationship between FDG- and MRI-based brain age 837 gap.

838
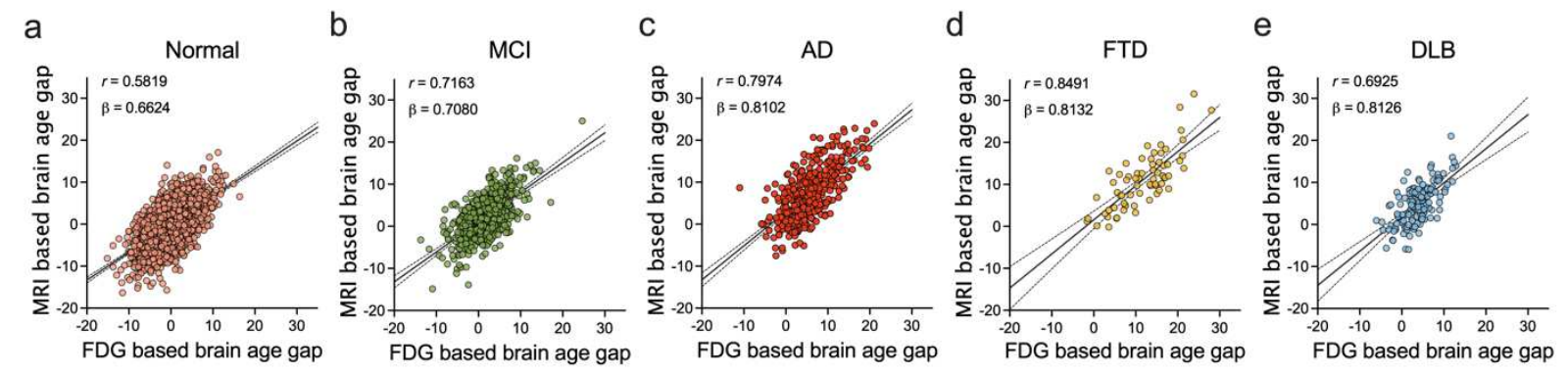

841 Supplementary figure 4. Relationship between FDG- and MRI-based brain age

842 gap. a, CU. b, MCl. c, AD. d, FTD. e, DLB. The black solid line and dotted lines in each

843 figure represent a regression line and its $95 \%$ confidence bands, respectively. $r$

844 indicates Pearson's correlation coefficient. 


\section{Supplementary table 1. Demographics of ADNI dataset.}

\begin{tabular}{cccc} 
Characteristic & \multicolumn{3}{c}{ Clinical Diagnosis } \\
& Normal & MCI & AD \\
\hline N & 330 & 647 & 255 \\
Total time points, $n(\%)$ & & & \\
1 & $208(63.03)$ & $443(68.47)$ & $227(89.02)$ \\
2 & $120(36.36)$ & $170(26.28)$ & $28(10.98)$ \\
3 & $2(0.61)$ & $34(5.26)$ & \\
Age, years & & & \\
Median (IQR) & $73(6978)$ & $74(6879)$ & $76(7181)$ \\
Min Max & 5696 & 5594 & 5696 \\
Male sex, n (\%) & $152(46.06)$ & $356(55.02)$ & $145(56.86)$ \\
\hline & $16(1518)$ & $16(1418)$ & $16(1418)$
\end{tabular}

848

849 
850 Supplementary table 2. Summary table of model performance.

\begin{tabular}{ccccc} 
Modality & Dataset & Val. MAE (yrs) & $\begin{array}{c}\text { Uncorrected } \\
\text { test MAE (yrs) }\end{array}$ & $\begin{array}{c}\text { Corrected } \\
\text { test MAE (yrs) }\end{array}$ \\
\hline \multirow{2}{*}{ FDG } & Mayo & $3.4558 \pm 0.1121$ & $3.4333 \pm 0.0545$ & $3.0755 \pm 0.1401$ \\
& Mayo model to ADNI & & 3.5097 & 2.8942 \\
& Mayo + ADNI & $3.0450 \pm 0.1360$ & $2.9943 \pm 0.1472$ & $2.7383 \pm 0.1091$ \\
\hline \multirow{2}{*}{ MRI } & Mayo & $4.1438 \pm 0.2012$ & $4.2055 \pm 0.2241$ & $3.4868 \pm 0.1631$ \\
& Mayo model to ADNI & & 4.2092 & 3.5766 \\
& Mayo + ADNI & $3.4886 \pm 0.1764$ & $3.5712 \pm 0.2010$ & $3.1029 \pm 0.2107$
\end{tabular}

851

852 


\section{Supplementary table 3. Data split strategy comparison}

\begin{tabular}{ccccc} 
Modality & Strategy & Val. MAE (yrs) & $\begin{array}{c}\text { Uncorrected } \\
\text { Test MAE (yrs) }\end{array}$ & $\begin{array}{c}\text { Corrected } \\
\text { Test MAE (yrs) }\end{array}$ \\
\hline & Option 1 & $3.4558 \pm 0.1121$ & $3.4333 \pm 0.0545$ & $3.0755 \pm 0.1401$ \\
FDG & Option 2 & $2.8381 \pm 0.0820$ & $2.8161 \pm 0.0581$ & $2.5773 \pm 0.0791$ \\
& Option 3 & $2.7197 \pm 0.0609$ & $3.2983 \pm 0.1221$ & $3.0606 \pm 0.1489$ \\
& Option 5 & $3.4094 \pm 0.0977$ & $3.4227 \pm 0.1717$ & $3.0822 \pm 0.1515$ \\
\hline & Option 1 & $4.1438 \pm 0.2012$ & $4.2055 \pm 0.2241$ & $3.4868 \pm 0.1631$ \\
& Option 2 & $3.4013 \pm 0.0789$ & $3.4101 \pm 0.0556$ & $2.9606 \pm 0.1152$ \\
MRI & Option 3 & $3.1033 \pm 0.1384$ & $3.8923 \pm 0.1896$ & $3.3339 \pm 0.0870$ \\
& Option 4 & $3.9168 \pm 0.1332$ & $4.0508 \pm 0.1326$ & $3.4393 \pm 0.1612$ \\
& Option 5 & $4.0204 \pm 0.1145$ & $4.0417 \pm 0.0999$ & $3.4701 \pm 0.1333$
\end{tabular}

854

855 
856 Supplementary table 4. Association of brain age gap with cognitive scores.

\begin{tabular}{cccccc} 
Modality & Cognitive test & Brain age gap correlation & $\mathbf{9 5 \%} \mathbf{C l}$ & $\mathbf{P}$ Value & $\mathbf{R}^{\mathbf{2}}$ \\
\hline \multirow{2}{*}{ FDG } & MMSE & -0.3870 & -0.4289 to -0.3434 & $<0.0001$ & 0.1498 \\
& Kokmen Short test & -0.3762 & -0.4190 to -0.3318 & $<0.0001$ & 0.1415 \\
& CDR sum of box & 0.3886 & 0.3460 to 0.4296 & $<0.0001$ & 0.1510 \\
\hline & MMSE & -0.3612 & -0.4041 to -0.3167 & $<0.0001$ & 0.1305 \\
MRI & Kokmen Short test & -0.3523 & -0.3960 to -0.3070 & $<0.0001$ & 0.1241 \\
& CDR sum of box & 0.3705 & 0.3272 to 0.4122 & $<0.0001$ & 0.1373
\end{tabular}

857

858 